\title{
Supplementary information: Complete mesoscopic parametrization of single LNA modifications in DNA applied to oncogene probe design
}

\author{
Izabela Ferreira $^{\mathrm{a}, \mathrm{b}}$, Sofie Slott ${ }^{\mathrm{c}}$, Kira Astakhova ${ }^{\mathrm{c}}$, Gerald Weber $^{\mathrm{a}, *}$ \\ ${ }^{a}$ Departamento de Física, Universidade Federal de Minas Gerais, Belo Horizonte-MG, Brazil. \\ ${ }^{b}$ Programa Interunidades de Pós-Graduação em Bioinformática, Universidade Federal de Minas Gerais, Belo \\ Horizonte-MG, Brazil \\ ${ }^{c}$ Department of Chemistry, Technical University of Denmark, Kemitorvet, Bygning 207, 2800 Kgs. Lyngby, Denmark
}

Supplementary Tab. S1 shows the number of modified and non-modified NN. Supplementary Tab. S2 shows the sequences used in the minimization and their respective measured and predicted temperature. Supplementary Tab. S3 shows the measured and predicted melting temperatures for the validation set of sequences containing LNA tandem modifications. Supplementary Tab. S4 shows a summary of the oncogenes used for the probe design and their respective human assemblies. Supplementary Tabs.S5-S11 show the melting temperature predictions for BRAF, EGFR and KRAS variants, respectively. Supplementary Tab.S12 shows the MALDI-MS and purities of the measured probes. Figs. S1-S14 show the HPLC and MALDI-MS of the measured probes. Figs. S15-S20 show the representative $T_{m}$ curve of the measured probes. Figs. S21-S24 show the average opening profiles for the LNA modified probes measured in this study. Fig. S25 shows the calculated configurational entropy $S_{y}$ comparing an unmodified DNA sequence to LNA modifications.

\footnotetext{
${ }^{*}$ Lead author

Email addresses: izabelaferreira13@gmail.com (Izabela Ferreira), sof slo@kemi.dtu.dk (Sofie Slott), kiraas@kemi.dtu.dk (Kira Astakhova), gweberbh@gmail.com (Gerald Weber)
} 
Table S1: Number of occurrences of base pairs and NN pairs contained in the final minimized dataset of Refs. [1-3]. LNA modifications are preceded by a plus sign and marked in bold.

\begin{tabular}{lrlrlrrrrr}
\hline \hline canonical & occurrences & modified & occurrences & modified & occurrences & modified & occurrences & modified & occurrences \\
\hline ATpAT & 253 & A+TpAT & 28 & ATpA+T & 26 & ATp+AT & 18 & TApT+A & \\
ATpTA & 155 & A+TpTA & 27 & ATpT+A & 27 & & & \\
TApAT & 164 & TAp+AT & 20 & +TApAT & 21 & & & \\
ATpCG & 386 & A+TpCG & 18 & ATpC+G & 27 & ATp+CG & 18 & GCpT+A & \\
ATpGC & 345 & A+TpGC & 24 & ATpG+C & 30 & ATp+GC & 12 & CGpT+A $+\mathbf{A}$ & 18 \\
CGpAT & 348 & CGpA+T & 31 & CGp+AT & 18 & C+GpAT & 25 & TApG+C & 19 \\
GCpAT & 360 & GCpA+T & 19 & GCp+AT & 22 & G+CpAT & 30 & +GCpAT & 17 \\
CGpCG & 287 & CGpC+G & 16 & CGp+CG & 19 & C+GpCG & 26 & GCpG+C & \\
CGpGC & 191 & CGpG+C & 21 & CGp+GC & 20 & & & \\
GCpCG & 195 & GCpC+G & 22 & GCp+CG & 21 & & & \\
\hline \hline
\end{tabular}


Table S2: Sequences used for the minimization procedure. Shown are the experimental temperatures $T_{i}$ and corresponding predictions $T_{i}^{\prime}$. All temperatures are in ${ }^{\circ} \mathrm{C}$. Also shown are total squared difference $\chi 2$ and the average difference of predicted and measured temperatures $\langle\Delta T\rangle$, Eqs. (6) and (8) in the main text. LNA modifications are preceded by a plus sign and marked in bold.

\begin{tabular}{|c|c|c|}
\hline $5^{\prime} \rightarrow 3^{\prime} / 3^{\prime} \rightarrow 5^{\prime}$ & $T_{i}\left({ }^{\circ} \mathrm{C}\right)$ & $T_{i}^{\prime}\left({ }^{\circ} \mathrm{C}\right)$ \\
\hline 1 CACGGCTC GTGCCGAG [1] & 40.0 & 40.3 \\
\hline 2 GGTGCCAA CCACGGTT [1] & 36.1 & 35.8 \\
\hline 3 TGCACGCTA ACGTGCGAT [1] & 45.4 & 42.8 \\
\hline 4 ACGTCTTCG TGCAGAAGC [1] & 39.7 & 39.7 \\
\hline 5 GCAGGTCTGC CGTCCAGACG [1] & 52.0 & 50.7 \\
\hline 6 GTCGAACAGC CAGCTTGTCG [1] & 49.5 & 46.2 \\
\hline 7 CTAAATAGCG GATTTATCGC [1] & 38.7 & 37.6 \\
\hline 8 GGACCTCGAC CCTG+GAGCTG [3] & 57.7 & 56.1 \\
\hline 9 GGACCTCGAC CCTGG+AGCTG [3] & 57.2 & 56.9 \\
\hline 10 GGACCTCGAC CCTGGAGCTG [3] & 50.9 & 51.7 \\
\hline 11 GGACCTCGAC CCT+GGAGCTG [3] & 55.2 & 56.6 \\
\hline 12 TTCATAGCCGT AA+GTATCGGCA [3] & 51.0 & 52.1 \\
\hline 13 TTCATAGCCGT AAGT+ATCGGCA [3] & 51.8 & 51.2 \\
\hline 14 TTCATAGCCGT AAGTAT+CGGCA [3] & 57.4 & 55.1 \\
\hline 15 TTCATAGCCGT AAGTATCGGCA [3] & 50.1 & 49.0 \\
\hline 16 TTCATAGCCGT AAGTA+TCGGCA [3] & 58.6 & 54.8 \\
\hline 17 TTCATAGCCGT AAG+TATCGGCA [3] & 53.1 & 54.2 \\
\hline 18 TTCATAGCCGT AAGTATC+GGCA [3] & 54.0 & 52.3 \\
\hline 19 TTGGGAGTAGC AACCCTCATCG [1] & 48.3 & 50.9 \\
\hline 20 ACTGGCATCTG TGACCGTAGAC [1] & 51.2 & 50.2 \\
\hline 21 CTATTGGCGAC GATAACCGCTG [3] & 52.4 & 50.2 \\
\hline 22 CTATTGGCGAC GATAA+CCGCTG [3] & 56.1 & 54.6 \\
\hline 23 ACAAGCGACTC TGTTCGCTGAG [1] & 52.5 & 50.0 \\
\hline 24 CTACGCATTCC GATGCG+TAAGG [3] & 54.2 & 55.3 \\
\hline 25 CTACGCATTCC GAT+GCGTAAGG [3] & 52.4 & 53.1 \\
\hline 26 CTACGCATTCC GATGCGTA+AGG [3] & 52.7 & 54.2 \\
\hline 27 CTACGCATTCC GATGCGTAAGG [3] & 50.1 & 50.2 \\
\hline 28 CTACGCATTCC GATGC+GTAAGG [3] & 52.1 & 53.5 \\
\hline 29 GTAGCAGGAGT CATCGTCCTCA [1] & 49.1 & 49.8 \\
\hline 30 CGCTGTTACGC GCGACAATGCG [1] & 53.4 & 53.7 \\
\hline 31 CCATTGCTACC GGTAACGATGG [1] & 48.9 & 49.5 \\
\hline 32 GTAGCGATGTA CATCGCTACAT [1] & 48.9 & 47.6 \\
\hline 33 ATTTGACTCAG TAAACTGAGTC [1] & 43.5 & 40.8 \\
\hline 34 GTGGATCTTTA CACCTAGAAAT [1] & 43.7 & 42.4 \\
\hline 35 GTATCAAGTCT CATAGT+TCAGA [3] & 47.2 & 46.5 \\
\hline 36 GTATCAAGTCT CATAGTTCAGA [3] & 43.9 & 41.2 \\
\hline 37 GTATCAAGTCT CATAGTT+CAGA [3] & 49.9 & 49.4 \\
\hline 38 GTATCAAGTCT CATAG+TTCAGA [3] & 48.2 & 45.3 \\
\hline 39 CTGAAGTCCGC GACTTC+AGGCG [3] & 59.7 & 58.3 \\
\hline 40 CTGAAGTCCGC GACTTCA+GGCG [3] & 59.7 & 58.3 \\
\hline
\end{tabular}

Continued on next page 
Table S2 - Continued from previous page

\begin{tabular}{|c|c|c|}
\hline $5^{\prime} \rightarrow 3^{\prime} / 3^{\prime} \rightarrow 5^{\prime}$ & $T_{i}\left({ }^{\circ} \mathrm{C}\right)$ & $T_{i}^{\prime}\left({ }^{\circ} \mathrm{C}\right)$ \\
\hline 41 CTGAAGTCCGC GACTTCAG+GCG [3] & 57.2 & 57.5 \\
\hline 42 CTGAAGTCCGC GACTTCAGGCG [3] & 54.9 & 53.5 \\
\hline 43 CTGAAGTCCGC GACTT+CAGGCG [3] & 61.4 & 59.6 \\
\hline 44 CGGTTGTGGCG GCCAACACCGC [1] & 57.8 & 57.5 \\
\hline 45 TGCGGATAAGT ACGCCT+ATTCA [3] & 51.6 & 51.2 \\
\hline 46 TGCGGATAAGT ACGCCTATTCA [3] & 50.7 & 49.0 \\
\hline 47 TGCGGATAAGT ACGCCTAT+TCA [3] & 52.5 & 53.8 \\
\hline 48 ATCTATCCGGC TAGATAGGCCG [1] & 50.9 & 52.9 \\
\hline 49 CGAACGTCTAT GCTTGCAGATA [1] & 46.2 & 47.2 \\
\hline 50 CCTGCGATGAC GGACGCTA+CTG [3] & 60.0 & 59.5 \\
\hline 51 CCTGCGATGAC GGACGCT+ACTG [3] & 56.6 & 56.9 \\
\hline 52 CCTGCGATGAC GGACGC+TACTG [3] & 58.8 & 57.7 \\
\hline 53 CCTGCGATGAC GGAC+GCTACTG [3] & 56.6 & 57.5 \\
\hline 54 CCTGCGATGAC GGACGCTACTG [3] & 54.9 & 54.3 \\
\hline 55 CCTGCGATGAC GGACG+CTACTG [3] & 59.8 & 58.8 \\
\hline 56 CTAACGGATGC GA+TTGCCTACG [3] & 53.6 & 54.1 \\
\hline 57 CTAACGGATGC GATTGCCTACG [3] & 50.2 & 50.2 \\
\hline 58 CTAACGGATGC GATTG+CCTACG [3] & 55.6 & 54.0 \\
\hline 59 CTAACGGATGC GATTGC+CTACG [3] & 55.2 & 53.1 \\
\hline 60 CTAACGGATGC GATTGCC+TACG [3] & 53.9 & 53.8 \\
\hline 61 CTAACGGATGC GAT+TGCCTACG [3] & 53.7 & 54.0 \\
\hline 62 CTAACGGATGC GATT+GCCTACG [3] & 54.0 & 53.1 \\
\hline 63 TTGCTCGATGT AACGAGCTACA [1] & 50.3 & 48.5 \\
\hline 64 GGAACAAGATGC CCTTG+TTCTACG [3] & 56.7 & 55.1 \\
\hline 65 GGAACAAGATGC CCTTGT+TCTACG [3] & 54.9 & 56.1 \\
\hline 66 GGAACAAGATGC CCTTGTT+CTACG [3] & 57.4 & 56.6 \\
\hline 67 GGAACAAGATGC CCTTGTTCTACG [3] & 52.4 & 51.7 \\
\hline 68 GGAACAAGATGC CCTT+GTTCTACG [3] & 56.1 & 54.3 \\
\hline 69 GGTCCTTGCTTGGTG CCAGGAACGAACCAC [2] & 67.8 & 65.5 \\
\hline 70 GGTCCTTTCTTGGTG CCAGGAAAGAACCAC [2] & 65.3 & 62.8 \\
\hline 71 GGTCCTTCCTTGGTG CCAGGAAGGAACCAC [2] & 67.4 & 65.8 \\
\hline 72 GGTCCTTACTTGGTG CCAGGAATGAACCAC [2] & 63.6 & 62.8 \\
\hline 73 ACGACCAGAGTTACAG TGCTGGTCTC+AATGTC [3] & 68.1 & 67.4 \\
\hline 74 ACGACCAGAGTTACAG TGCTGGTCTCA+ATGTC [3] & 66.8 & 65.8 \\
\hline 75 ACGACCAGAGTTACAG TGCTGGTCTCAATGTC [3] & 65.5 & 64.6 \\
\hline 76 ACGACCAGAGTTACAG TGCTGGTCTCAA+TGTC [3] & 68.3 & 67.5 \\
\hline 77 ACGACCAGAGTTACAG TGCTG+GTCTCAATGTC [3] & 69.2 & 67.5 \\
\hline 78 ACGACCAGAGTTACAG TGCT+GGTCTCAATGTC [3] & 68.5 & 67.8 \\
\hline 79 ACGACCAGAGTTACAG TGCTGGT+CTCAATGTC [3] & 69.0 & 67.9 \\
\hline 80 CACGG+CTC GTGCCGAG [1] & 47.5 & 45.6 \\
\hline 81 GGTG+CCAA CCACGGTT [1] & 41.5 & 39.1 \\
\hline 82 GGT+GCCAA CCACGGTT [1] & 38.4 & 39.8 \\
\hline 83 GGTGC+CAA CCACGGTT [1] & 39.1 & 40.6 \\
\hline 84 CA+CGGCTC GTGCCGAG [1] & 46.8 & 47.2 \\
\hline
\end{tabular}

Continued on next page 
Table S2 - Continued from previous page

\begin{tabular}{|c|c|c|}
\hline $5^{\prime} \rightarrow 3^{\prime} / 3^{\prime} \rightarrow 5^{\prime}$ & $T_{i}\left({ }^{\circ} \mathrm{C}\right)$ & $T_{i}^{\prime}\left({ }^{\circ} \mathrm{C}\right)$ \\
\hline 85 CAC+GGCTC GTGCCGAG [1] & 43.6 & 44.7 \\
\hline 86 GG+TGCCAA CCACGGTT [1] & 42.2 & 41.9 \\
\hline 87 CACG+GCTC GTGCCGAG [1] & 47.3 & 46.1 \\
\hline 88 ACGTCT+TCG TGCAGAAGC [1] & 42.1 & 42.2 \\
\hline 89 ACGT+CTTCG TGCAGAAGC [1] & 46.9 & 44.9 \\
\hline 90 TGCAC+GCTA ACGTGCGAT [1] & 47.5 & 46.1 \\
\hline 91 TGC+ACGCTA ACGTGCGAT [1] & 46.8 & 47.3 \\
\hline 92 AC+GTCTTCG TGCAGAAGC [1] & 40.8 & 43.3 \\
\hline 93 TG+CACGCTA ACGTGCGAT [1] & 47.9 & 47.1 \\
\hline 94 ACGTC+TTCG TGCAGAAGC [1] & 43.2 & 45.6 \\
\hline 95 ACG+TCTTCG TGCAGAAGC [1] & 42.9 & 43.4 \\
\hline 96 CTAAA+TAGCG GATTTATCGC [1] & 41.8 & 44.3 \\
\hline 97 CTAA+ATAGCG GATTTATCGC [1] & 40.0 & 42.4 \\
\hline 98 GGACCT+CGAC CCTGGAGCTG [3] & 55.7 & 55.7 \\
\hline 99 CT+AAATAGCG GATTTATCGC [1] & 37.7 & 40.1 \\
\hline 100 GC+AGGTCTGC CGTCCAGACG [1] & 55.4 & 54.7 \\
\hline 101 GTCGAA+CAGC CAGCTTGTCG [1] & 53.9 & 54.8 \\
\hline 102 GTCGA+ACAGC CAGCTTGTCG [1] & 51.5 & 51.6 \\
\hline 103 TATTAAGCGACCACACATAA ATAATTCGCTGGTGTGTATT [1] & 69.1 & 67.2 \\
\hline 104 GTCG+AACAGC CAGCTTGTCG [1] & 51.3 & 50.8 \\
\hline 105 GCAG+GTCTGC CGTCCAGACG [1] & 55.9 & 55.4 \\
\hline 106 GGA+CCTCGAC CCTGGAGCTG [3] & 57.2 & 56.9 \\
\hline 107 GCA+GGTCTGC CGTCCAGACG [1] & 58.3 & 56.8 \\
\hline 108 GTC+GAACAGC CAGCTTGTCG [1] & 48.3 & 50.1 \\
\hline 109 ATTATGCTCCAATCATGTCG TAATACGAGGTTAGTACAGC [1] & 68.1 & 68.5 \\
\hline 110 GGAC+CTCGAC CCTGGAGCTG [3] & 55.3 & 55.9 \\
\hline 111 GGACC+TCGAC CCTGGAGCTG [1] & 53.3 & 55.8 \\
\hline 112 CTA+AATAGCG GATTTATCGC [1] & 41.1 & 42.2 \\
\hline 113 CTA+ACGGATGC GATTGC+CTACG [3] & 61.9 & 59.0 \\
\hline 114 CTA+ACGGATGC GATTG+CCTACG [3] & 60.1 & 57.8 \\
\hline 115 CTA+ACGGATGC GATTGCCTACG [3] & 52.7 & 55.0 \\
\hline 116 CCTGC+GATGAC GGACGCTACTG [3] & 57.5 & 57.4 \\
\hline 117 CTAAC+GGATGC GATTGCCTACG [3] & 53.8 & 53.2 \\
\hline 118 CTAAC+GGATGC GAT+TGCCTACG [3] & 56.1 & 58.0 \\
\hline 119 CTAAC+GGATGC GATTGCC+TACG [3] & 60.0 & 57.7 \\
\hline 120 CTACGCA+TTCC GATGCGTAAGG [3] & 53.2 & 54.6 \\
\hline 121 CTGA+AGTCCGC GACTTCA+GGCG [3] & 65.4 & 62.6 \\
\hline 122 CTGA+AGTCCGC GACTTCAGGCG [3] & 60.5 & 58.1 \\
\hline 123 AT+TTGACTCAG TAAACTGAGTC [1] & 45.1 & 43.9 \\
\hline 124 TTC+ATAGCCGT AAGTA+TCGGCA [3] & 58.8 & 57.4 \\
\hline 125 TTC+ATAGCCGT AAGTATCGGCA [3] & 52.4 & 51.8 \\
\hline 126 TTC+ATAGCCGT AAGTATC+GGCA [3] & 55.2 & 56.3 \\
\hline 127 TTC+ATAGCCGT AAGTAT+CGGCA [3] & 58.4 & 55.6 \\
\hline 128 TTGGGAG+TAGC AACCCTCATCG [1] & 53.2 & 55.7 \\
\hline
\end{tabular}

Continued on next page 
Table S2 - Continued from previous page

\begin{tabular}{|c|c|c|}
\hline $5^{\prime} \rightarrow 3^{\prime} / 3^{\prime} \rightarrow 5^{\prime}$ & $T_{i}\left({ }^{\circ} \mathrm{C}\right)$ & $T_{i}^{\prime}\left({ }^{\circ} \mathrm{C}\right)$ \\
\hline 129 GTGGATCT+TTA CACCTAGAAAT [1] & 45.1 & 45.5 \\
\hline 130 CCTGCGA+TGAC GGACGCTACTG [3] & 59.1 & 59.3 \\
\hline 131 CGGTT+GTGGCG GCCAACACCGC [1] & 60.0 & 60.1 \\
\hline 132 TTGCTCGA+TGT AACGAGCTACA [1] & 54.2 & 53.8 \\
\hline 133 ATTT+GACTCAG TAAACTGAGTC [1] & 45.2 & 44.4 \\
\hline 134 CGCTGTT+ACGC GCGACAATGCG [1] & 55.0 & 56.6 \\
\hline 135 CTG+AAGTCCGC GACTTCAGGCG [3] & 59.7 & 57.5 \\
\hline 136 CTG+AAGTCCGC GACTTC+AGGCG [3] & 64.3 & 61.7 \\
\hline 137 CGGTTGTG+GCG GCCAACACCGC [1] & 60.8 & 61.5 \\
\hline 138 ATCTA+TCCGGC TAGATAGGCCG [1] & 52.6 & 56.4 \\
\hline 139 CTAA+CGGATGC GATTGCC+TACG [3] & 60.4 & 60.4 \\
\hline 140 CTAA+CGGATGC GATTGCCTACG [3] & 54.2 & 55.0 \\
\hline 141 CTAA+CGGATGC GATTGC+CTACG [3] & 61.9 & 59.7 \\
\hline 142 CC+TGCGATGAC GGACGCTACTG [3] & 58.4 & 59.4 \\
\hline 143 CG+GTTGTGGCG GCCAACACCGC [1] & 61.4 & 61.3 \\
\hline 144 CGAACGTC+TAT GCTTGCAGATA [1] & 49.9 & 53.1 \\
\hline 145 CCAT+TGCTACC GGTAACGATGG [1] & 51.9 & 53.2 \\
\hline 146 CTGAA+GTCCGC GACTTCA+GGCG [3] & 64.3 & 62.4 \\
\hline 147 CTGAA+GTCCGC GACTTCAGGCG [3] & 59.3 & 58.0 \\
\hline 148 CTGAA+GTCCGC GACTTCAG+GCG [3] & 62.8 & 61.2 \\
\hline 149 CTACGC+ATTCC GATGCGTA+AGG [3] & 57.6 & 56.9 \\
\hline 150 CTACGC+ATTCC GATGCGTAAGG [3] & 51.2 & 52.9 \\
\hline 151 ATCTATC+CGGC TAGATAGGCCG [1] & 53.0 & 54.4 \\
\hline 152 GT+AGCAGGAGT CATCGTCCTCA [1] & 50.1 & 52.9 \\
\hline 153 GT+GGATCTTTA CACCTAGAAAT [1] & 46.0 & 47.1 \\
\hline 154 TTGGG+AGTAGC AACCCTCATCG [1] & 54.3 & 55.9 \\
\hline 155 GTAGC+GATGTA CATCGCTACAT [1] & 51.3 & 51.3 \\
\hline 156 GTGGA+TCTTTA CACCTAGAAAT [1] & 45.6 & 46.3 \\
\hline 157 GTAGCAGG+AGT CATCGTCCTCA [1] & 52.6 & 55.0 \\
\hline 158 CCATTG+CTACC GGTAACGATGG [1] & 53.8 & 55.9 \\
\hline 159 AC+TGGCATCTG TGACCGTAGAC [1] & 53.4 & 55.7 \\
\hline 160 ATTTGACT+CAG TAAACTGAGTC [1] & 47.0 & 48.2 \\
\hline 161 ACTGGCAT+CTG TGACCGTAGAC [1] & 54.8 & 55.5 \\
\hline 162 CTAACGG+ATGC GATT+GCCTACG [3] & 54.5 & 56.9 \\
\hline 163 CTAACGG+ATGC GATTGCCTACG [3] & 53.4 & 54.3 \\
\hline 164 CTAACGG+ATGC GATTG+CCTACG [3] & 57.5 & 55.2 \\
\hline 165 AC+AAGCGACTC TGTTCGCTGAG [1] & 52.5 & 52.6 \\
\hline 166 CTATTGG+CGAC GATAA+CCGCTG [3] & 59.1 & 59.2 \\
\hline 167 CTATTGG+CGAC GATAACCGCTG [3] & 56.7 & 53.4 \\
\hline 168 TT+GCTCGATGT AACGAGCTACA [1] & 51.9 & 51.7 \\
\hline 169 CGCT+GTTACGC GCGACAATGCG [1] & 56.3 & 56.4 \\
\hline 170 CT+GAAGTCCGC GACTTC+AGGCG [3] & 61.0 & 60.6 \\
\hline 171 CT+GAAGTCCGC GACTT+CAGGCG [3] & 63.3 & 60.3 \\
\hline 172 CT+GAAGTCCGC GACTTCAGGCG [3] & 56.0 & 56.6 \\
\hline
\end{tabular}

Continued on next page 
Table S2 - Continued from previous page

\begin{tabular}{|c|c|c|}
\hline $5^{\prime} \rightarrow 3^{\prime} / 3^{\prime} \rightarrow 5^{\prime}$ & $T_{i}\left({ }^{\circ} \mathrm{C}\right)$ & $T_{i}^{\prime}\left({ }^{\circ} \mathrm{C}\right)$ \\
\hline 173 TTGCT+CGATGT AACGAGCTACA [1] & 53.4 & 53.4 \\
\hline 174 CTAACG+GATGC GATT+GCCTACG [3] & 56.7 & 54.0 \\
\hline 175 CTAACG+GATGC GAT+TGCCTACG [3] & 57.4 & 58.8 \\
\hline 176 CTAACG+GATGC GATTGCCTACG [3] & 55.3 & 53.9 \\
\hline 177 ACTGG+CATCTG TGACCGTAGAC [1] & 58.0 & 55.3 \\
\hline 178 CTACG+CATTCC GATGCGTAAGG [3] & 56.4 & 54.8 \\
\hline 179 CTACG+CATTCC GAT+GCGTAAGG [3] & 59.3 & 59.0 \\
\hline 180 CCTGCG+ATGAC GGACGCTACTG [3] & 57.9 & 58.4 \\
\hline 181 TTCAT+AGCCGT AAGTATCGGCA [3] & 53.0 & 52.1 \\
\hline 182 TTCAT+AGCCGT AAGTATC+GGCA [3] & 59.9 & 56.6 \\
\hline 183 TTCAT+AGCCGT AAG+TATCGGCA [3] & 55.2 & 57.1 \\
\hline 184 CCA+TTGCTACC GGTAACGATGG [1] & 51.5 & 54.0 \\
\hline 185 GCTACTC+CCAA CGATGAGGGTT [1] & 52.3 & 54.3 \\
\hline 186 CC+ATTGCTACC GGTAACGATGG [1] & 48.3 & 52.3 \\
\hline 187 CGC+TGTTACGC GCGACAATGCG [1] & 58.7 & 58.7 \\
\hline 188 ATTTG+ACTCAG TAAACTGAGTC [1] & 46.6 & 46.6 \\
\hline 189 GTA+TCAAGTCT CATAGT+TCAGA [3] & 51.8 & 50.1 \\
\hline 190 GTA+TCAAGTCT CATAGTTCAGA [3] & 47.2 & 45.2 \\
\hline 191 CGAAC+GTCTAT GCTTGCAGATA [1] & 49.0 & 50.4 \\
\hline 192 CCT+GCGATGAC GGACGCTACTG [3] & 56.2 & 57.3 \\
\hline 193 TTCA+TAGCCGT AAGTATCGGCA [3] & 54.5 & 54.5 \\
\hline 194 TTCA+TAGCCGT AAGTATC+GGCA [3] & 60.9 & 56.9 \\
\hline 195 TTCA+TAGCCGT AAGTAT+CGGCA [3] & 63.1 & 59.9 \\
\hline 196 GTA+GCGATGTA CATCGCTACAT [1] & 50.9 & 52.9 \\
\hline 197 TGCGGA+TAAGT ACGCCTATTCA [3] & 52.9 & 54.5 \\
\hline 198 TGCGGA+TAAGT ACGCCTAT+TCA [3] & 59.0 & 58.9 \\
\hline 199 ACAAGCGA+CTC TGTTCGCTGAG [1] & 57.5 & 56.7 \\
\hline 200 TTCATA+GCCGT AAG+TATCGGCA [3] & 55.8 & 56.5 \\
\hline 201 TTCATA+GCCGT AAGTATCGGCA [3] & 52.6 & 53.1 \\
\hline 202 TTCATA+GCCGT AAGT+ATCGGCA [3] & 55.1 & 55.8 \\
\hline 203 ACAAG+CGACTC TGTTCGCTGAG [1] & 57.1 & 53.2 \\
\hline 204 TTGG+GAGTAGC AACCCTCATCG [1] & 52.6 & 55.4 \\
\hline 205 GT+ATCAAGTCT CATAGTTCAGA [3] & 46.8 & 43.6 \\
\hline 206 GT+ATCAAGTCT CATAG+TTCAGA [3] & 51.6 & 47.6 \\
\hline 207 GTATC+AAGTCT CATAGTTCAGA [3] & 46.2 & 44.2 \\
\hline 208 CCTG+CGATGAC GGACGCTACTG [3] & 59.6 & 60.2 \\
\hline 209 ATTTGA+CTCAG TAAACTGAGTC [1] & 49.1 & 48.9 \\
\hline 210 TTGGGAGT+AGC AACCCTCATCG [1] & 52.0 & 53.9 \\
\hline 211 TT+CATAGCCGT AAGTATCGGCA [3] & 54.7 & 54.6 \\
\hline 212 TT+CATAGCCGT AAGTA+TCGGCA [3] & 61.1 & 59.5 \\
\hline 213 TT+CATAGCCGT AAGT+ATCGGCA [3] & 60.1 & 57.3 \\
\hline 214 TT+CATAGCCGT AAGTAT+CGGCA [3] & 62.2 & 59.3 \\
\hline 215 CG+AACGTCTAT GCTTGCAGATA [1] & 48.9 & 51.4 \\
\hline 216 ATCTAT+CCGGC TAGATAGGCCG [1] & 53.1 & 56.6 \\
\hline
\end{tabular}

Continued on next page 
Table S2 - Continued from previous page

\begin{tabular}{|c|c|c|}
\hline $5^{\prime} \rightarrow 3^{\prime} / 3^{\prime} \rightarrow 5^{\prime}$ & $T_{i}\left({ }^{\circ} \mathrm{C}\right)$ & $T_{i}^{\prime}\left({ }^{\circ} \mathrm{C}\right)$ \\
\hline 217 TGCGGATA+AGT ACGCCT+ATTCA [3] & 55.2 & 55.8 \\
\hline 218 TGCGGATA+AGT ACGCCTATTCA [3] & 54.6 & 53.8 \\
\hline 219 CTA+CGCATTCC GATGCG+TAAGG [3] & 61.3 & 58.1 \\
\hline 220 CTA+CGCATTCC GATGC+GTAAGG [3] & 60.6 & 59.0 \\
\hline 221 CTA+CGCATTCC GATGCGTAAGG [3] & 54.6 & 55.0 \\
\hline 222 GTAG+CGATGTA CATCGCTACAT [1] & 53.6 & 53.8 \\
\hline 223 GTAGC+AGGAGT CATCGTCCTCA [1] & 51.9 & 53.6 \\
\hline 224 TTCATAG+CCGT AAGTA+TCGGCA [3] & 60.0 & 57.2 \\
\hline 225 TTCATAG+CCGT AAGTATCGGCA [3] & 55.1 & 53.4 \\
\hline 226 TTCATAG+CCGT AAGT+ATCGGCA [3] & 57.3 & 56.6 \\
\hline 227 TTGGGA+GTAGC AACCCTCATCG [1] & 52.9 & 55.5 \\
\hline 228 TT+GGGAGTAGC AACCCTCATCG [1] & 51.3 & 54.1 \\
\hline 229 CGCTG+TTACGC GCGACAATGCG [1] & 56.8 & 57.4 \\
\hline 230 CGCTGT+TACGC GCGACAATGCG [1] & 57.0 & 57.4 \\
\hline 231 ATC+TATCCGGC TAGATAGGCCG [1] & 54.8 & 58.5 \\
\hline 232 CCATT+GCTACC GGTAACGATGG [1] & 50.5 & 52.9 \\
\hline 233 ATCT+ATCCGGC TAGATAGGCCG [1] & 52.5 & 55.0 \\
\hline 234 TTG+GGAGTAGC AACCCTCATCG [1] & 53.1 & 56.2 \\
\hline 235 CTACGCAT+TCC GATGCG+TAAGG [3] & 56.7 & 57.2 \\
\hline 236 CTACGCAT+TCC GATGCGTAAGG [3] & 54.1 & 52.2 \\
\hline 237 GTAT+CAAGTCT CATAGTTCAGA [3] & 48.3 & 48.7 \\
\hline 238 GTAGCGA+TGTA CATCGCTACAT [1] & 54.7 & 52.9 \\
\hline 239 GGA+ACAAGATGC CCTTGTTCTACG [3] & 54.0 & 56.2 \\
\hline 240 GGAACAAG+ATGC CCTTGTTCTACG [3] & 54.9 & 55.7 \\
\hline 241 GGAACA+AGATGC CCTTGTTCTACG [3] & 55.9 & 56.1 \\
\hline 242 GGAAC+AAGATGC CCTTGTTCTACG [3] & 53.9 & 54.2 \\
\hline 243 GGAACAA+GATGC CCTTGTTCTACG [1] & 56.9 & 56.4 \\
\hline 244 CGGTCATCCACGTAGCCAGGTCA GCCAGTAGGTGCATCGGTCCAGT [2] & 77.7 & 75.1 \\
\hline 245 CGGTCATCAACGTAGCAAGGTCA GCCAGTAGTTGCATCGTTCCAGT [2] & 75.8 & 72.8 \\
\hline 246 CGGTCATCTACGTAGCTAGGTCA GCCAGTAGATGCATCGATCCAGT [2] & 74.1 & 73.0 \\
\hline 247 CGGTCATCGACGTAGCGAGGTCA GCCAGTAGCTGCATCGCTCCAGT [2] & 78.8 & 75.4 \\
\hline 248 GGTCCT+TGCTTGGTG CCAGGAACGAACCAC [2] & 66.2 & 67.9 \\
\hline 249 GGTCCTT+ACTTGGTG CCAGGAATGAACCAC [2] & 60.9 & 64.8 \\
\hline 250 GGTCCTTT+CTTGGTG CCAGGAAAGAACCAC [2] & 64.4 & 66.4 \\
\hline 251 GGTCCTTG+CTTGGTG CCAGGAACGAACCAC [2] & 67.4 & 69.8 \\
\hline 252 GGTCCTTA+CTTGGTG CCAGGAATGAACCAC [2] & 63.6 & 67.8 \\
\hline 253 GGTCCT+TTCTTGGTG CCAGGAAAGAACCAC [2] & 62.5 & 64.7 \\
\hline 254 GGTCCTT+CCTTGGTG CCAGGAAGGAACCAC [2] & 65.6 & 68.7 \\
\hline 255 GGTCCTT+TCTTGGTG CCAGGAAAGAACCAC [2] & 62.9 & 64.1 \\
\hline 256 GGTCCT+TCCTTGGTG CCAGGAAGGAACCAC [2] & 64.3 & 67.1 \\
\hline 257 GGTCCTTC+CTTGGTG CCAGGAAGGAACCAC [2] & 65.6 & 68.7 \\
\hline 258 GGTCCTT+GCTTGGTG CCAGGAACGAACCAC [2] & 65.1 & 67.9 \\
\hline 259 GGTCCT+TACTTGGTG CCAGGAATGAACCAC [2] & 62.0 & 65.4 \\
\hline 260 ACGACCAGAG+TTACAG TGCTGGTCTCAATGTC [3] & 68.1 & 66.8 \\
\hline
\end{tabular}

Continued on next page 
Table S2 - Continued from previous page

\begin{tabular}{|c|c|c|}
\hline $5^{\prime} \rightarrow 3^{\prime} / 3^{\prime} \rightarrow 5^{\prime}$ & $T_{i}\left({ }^{\circ} \mathrm{C}\right)$ & $T_{i}^{\prime}\left({ }^{\circ} \mathrm{C}\right)$ \\
\hline 261 ACG+ACCAGAGTTACAG TGCTGGTCTCAATGTC [3] & 67.5 & 67.8 \\
\hline 262 ACGACCAGAGT+TACAG TGCTGGTCTCAATGTC [3] & 68.4 & 66.9 \\
\hline 263 ACGACCAG+AGTTACAG TGCTGGTCTCAATGTC [3] & 69.5 & 67.8 \\
\hline 264 ACGACCAGA+GTTACAG TGCTGGTCTCAATGTC [3] & 67.9 & 67.4 \\
\hline 265 ACGAC+CAGAGTTACAG TGCTGGTCTCAATGTC [3] & 68.6 & 68.4 \\
\hline 266 AC+GACCAGAGTTACAG TGCTGGTCTCAATGTC [3] & 66.2 & 66.9 \\
\hline 267 ACGA+CCAGAGTTACAG TGCTGGTCTCAATGTC [3] & 69.3 & 68.6 \\
\hline 268 AT+TATGCTCCAATCATGTCG TAATACGAGGTTAGTACAGC [1] & 70.0 & 70.0 \\
\hline 269 ATTAT+GCTCCAATCATGTCG TAATACGAGGTTAGTACAGC [1] & 69.8 & 70.1 \\
\hline 270 ATTATGCT+CCAATCATGTCG TAATACGAGGTTAGTACAGC [1] & 71.2 & 70.5 \\
\hline 271 TATTAAGCGACCACACA+TAA ATAATTCGCTGGTGTGTATT [1] & 70.4 & 69.4 \\
\hline 272 TATTAAGCGAC+CACACATAA ATAATTCGCTGGTGTGTATT [1] & 71.0 & 69.8 \\
\hline 273 TATTAAGCGACCAC+ACATAA ATAATTCGCTGGTGTGTATT [1] & 70.2 & 68.8 \\
\hline 274 ATTATGCTCCAATC+ATGTCG TAATACGAGGTTAGTACAGC [1] & 69.8 & 69.6 \\
\hline 275 GGAC+CT+CGAC CCTGGAGCTG [3] & 61.2 & 57.4 \\
\hline 276 ATTATGCTCCA+ATCATGTCG TAATACGAGGTTAGTACAGC [1] & 69.9 & 70.0 \\
\hline 277 TA+TTAAGCGACCACACATAA ATAATTCGCTGGTGTGTATT [1] & 69.3 & 69.0 \\
\hline 278 TATTA+AGCGACCACACATAA ATAATTCGCTGGTGTGTATT [1] & 70.5 & 69.1 \\
\hline 279 ATTATGCTCCAATCATG+TCG TAATACGAGGTTAGTACAGC [1] & 71.0 & 69.7 \\
\hline 280 TATTAAGC+GACCACACATAA ATAATTCGCTGGTGTGTATT [1] & 69.9 & 68.8 \\
\hline 281 GGAACA+AG+ATGC CCTTGTTCTACG [3] & 59.3 & 59.8 \\
\hline 282 GGA+AC+AAGATGC CCTTGTTCTACG [3] & 58.0 & 58.5 \\
\hline 283 GGTCCT+TG+CTTGGTG CCAGGAACGAACCAC [2] & 69.5 & 71.3 \\
\hline 284 GGTCCT+TC+CTTGGTG CCAGGAAGGAACCAC [2] & 68.1 & 69.9 \\
\hline 285 GGTCCT+TA+CTTGGTG CCAGGAATGAACCAC [2] & 65.7 & 70.1 \\
\hline 286 GGTCCT+TT+CTTGGTG CCAGGAAAGAACCAC [2] & 65.8 & 68.2 \\
\hline $287 \mathbf{G + G T C}+\mathbf{C T T}+\mathbf{G C T}+\mathbf{T G G}+\mathbf{T G}$ CCAGGAACGAACCAC [2] & 76.4 & 74.8 \\
\hline 288 G+GTC+CTT+TCT+TGG+TG CCAGGAAAGAACCAC [2] & 73.3 & 74.3 \\
\hline 289 G+GTC+CTT+ACT+TGG+TG CCAGGAATGAACCAC [2] & 72.8 & 72.6 \\
\hline $290 \mathbf{G + G T C + C T T + C C T + T G G + T G ~ C C A G G A A G G A A C C A C ~ [ 2 ] ~}$ & 76.5 & 74.8 \\
\hline $291 \mathbf{G}+\mathbf{G T}+\mathbf{C C}+\mathbf{T T}+\mathbf{T C}+\mathbf{T T}+\mathbf{G G}+\mathbf{T G}$ CCAGGAAAGAACCAC [2] & 79.0 & 77.9 \\
\hline $292 \mathbf{G}+\mathbf{G T}+\mathbf{C C}+\mathbf{T T}+\mathbf{A C}+\mathbf{T T}+\mathbf{G G}+\mathbf{T G}$ CCAGGAATGAACCAC [2] & 79.7 & 76.8 \\
\hline $293 \mathbf{G + G T + C C + T T + G C + T T + G G + T G ~ C C A G G A A C G A A C C A C ~ [ 2 ] ~}$ & 83.5 & 78.3 \\
\hline $294 \mathbf{G + G T + C C + T T + C C + T T + G G + T G ~ C C A G G A A G G A A C C A C ~ [ 2 ] ~}$ & 83.3 & 79.6 \\
\hline$\langle\Delta T\rangle$ & & $1.48^{\circ} \mathrm{C}$ \\
\hline$\chi^{2}$ & & $1013.60^{\circ} \mathrm{C}^{2}$ \\
\hline
\end{tabular}


Table S3: Predicted temperatures for sequences bearing LNA tandem modifications from Ref.[2-4] using the optimized parameters from EE-DL minimization round. Also shown are total squared difference $\chi 2$ and the average difference of predicted and measured temperatures $\langle\Delta T\rangle$.

\begin{tabular}{lcc}
\hline \hline 5 $^{\prime} \rightarrow 3^{\prime} / 3^{\prime} \rightarrow$ 5 $^{\prime}$ & $T_{i}\left({ }^{\circ} \mathrm{C}\right)$ & $T_{i}^{\prime}\left({ }^{\circ} \mathrm{C}\right)$ \\
\hline \hline 1 GGACCTCGAC/CCT+G+GAGCTG [3] & 60.9 & 59.7 \\
2 TTCATAGCCGT/AA+G+TATCGGCA [4] & 57.9 & 55.1 \\
3 TTCATAGCCGT/AAGTATC+G+GCA [4] & 56.9 & 54.9 \\
4 TTCATAGCCGT/AAGT+A+TCGGCA [4] & 56.8 & 56.7 \\
5 TTCATAGCCGT/AAGTA+T+CGGCA [4] & 60.7 & 58.8 \\
6 CTACGCATTCC/GATGCGT+A+AGG [4] & 52.8 & 58.0 \\
7 CTAACGGATGC/GAT+T+GCCTACG [4] & 52.7 & 57.7 \\
8 CTAACGGATGC/GATTGC+C+TACG [4] & 57.6 & 56.5 \\
9 GGAACAAGATGC/CCTTG+T+TCTACG [3] & 59.2 & 60.4 \\
10 GGA+C+CTCGAC/CCTGGAGCTG [3] & 59.3 & 58.3 \\
11 CTA+A+CGGATGC/GATTGCCTACG [4] & 56.5 & 60.5 \\
12 TT+C+ATAGCCGT/AAGTATCGGCA [4] & 55.5 & 54.3 \\
13 CTA+C+GCATTCC/GATGCGTAAGG [4] & 58.2 & 58.4 \\
14 TTCATAG+C+CGT/AAGTATCGGCA [4] & 59.3 & 55.1 \\
15 TTCAT+A+GCCGT/AAGTATCGGCA [4] & 55.3 & 56.1 \\
16 CTAACG+G+ATGC/GATTGCCTACG [4] & 56.4 & 54.4 \\
17 CTAC+G+CATTCC/GATGCGTAAGG [4] & 56.9 & 57.0 \\
18 CTACGC+A+TTCC/GATGCGTAAGG [4] & 53.5 & 55.5 \\
19 CTACGCA+T+TCC/GATGCGTAAGG [4] & 57.0 & 57.7 \\
20 GGAAC+A+AGATGC/CCTTGTTCTACG [3] & 57.1 & 58.6 \\
21 GGTCCT+T+TCTTGGTG/CCAGGAAAGACCAC [2] & 64.3 & 66.8 \\
22 GGTCCT+T+GCTTGGTG/CCAGGAACGAACCAC [2] & 69.7 & 70.5 \\
23 GGTCCT+T+ACTTGGTG/CCAGGAATGAACCAC [2] & 65.6 & 67.4 \\
24 GGTCCT+T+CCTTGGTG/CCAGGAAGGAACCAC [2] & 67.7 & 71.0 \\
\hline \hline$\langle\Delta T\rangle$ & & $1.94{ }^{\circ} \mathrm{C}$ \\
$\chi^{2}$ & & $139.50{ }^{\circ} \mathrm{C}^{2}$ \\
\hline \hline
\end{tabular}


Table S4: Summary of the oncogenes used for the probe design and their respective human assemblies.

\begin{tabular}{llllll}
\hline \hline Oncogene & Gene ID & HGNC & Ensembl & MIM & GRCh38.p13 \\
\hline \hline BRAF & 673 & 1097 & ENSG00000157764 & 164757 & GCF_000001405.39 \\
$K R A S$ & 3845 & 6407 & ENSG00000133703 & 190070 & GCF_000001405.39 \\
$E G F R$ & 1956 & 3236 & ENSG00000146648 & 131550 & GCF_000001405.39 \\
\hline \hline
\end{tabular}

Table S5: Predicted temperatures for BRAF capture LNA modified probe at medium salt concentration using the calculated parameters at high salt concentration from EE-DL minimization and applying salt correction for DNA[5] sequences. LNA modifications are preceded by a plus sign and marked in bold. A cut-off was made in the tables to show only the 30 sequences with the highest and lowest temperature difference between the modified and nonmodified probe, the total number of predicted sequences was 1562. As comparative, we also show the $\Delta T_{m}$ of each modified sequence and its non-modified analog. The chosen probe is highlighted in yellow.

\begin{tabular}{l|l|l|l}
\hline$T_{m}$ & $\Delta T_{m}$ & Probe $\left(5^{\prime} \rightarrow 3^{\prime}\right)$ & ID \\
\hline 59.5693 & 0 & ATCGAGATTTCTCTGTAGCTA & BRAFcap-CTRL \\
\hline 65.6223 & 6.053 & AT+CGAGA+TTTCTCTGTAG+CTA & BRAFcap-M481 \\
65.5789 & 6.010 & ATCGA+GATTTCTCTGTAG+C+TA & BRAFcap-M982 \\
65.4673 & 5.898 & ATCGAGATTTCTCTG+TAG+C+TA & BRAFcap-M1532 \\
65.4294 & 5.860 & A+T+CGAGATTTCTCTGTA+GCTA & BRAFcap-M228 \\
65.4029 & 5.834 & ATCGAGATTT+CTC+TGTAG+CTA & BRAFcap-M1356 \\
65.3801 & 5.811 & ATCGAGATTT+CT+CTGTAG+CTA & BRAFcap-M1348 \\
65.3745 & 5.805 & A+T+CGAGATTTCTCTGTAGC+TA & BRAFcap-M230 \\
65.3631 & 5.794 & ATCGAGA+TTTCTCTGTAG+C+TA & BRAFcap-M1180 \\
65.327 & 5.758 & AT+CGAGATTTCTCTGTA+GC+TA & BRAFcap-M567 \\
65.2719 & 5.703 & AT+CGAGATTTC+T+CTGTAGCTA & BRAFcap-M521 \\
65.2643 & 5.695 & A+T+CGAGATTTCTC+TGTAGCTA & BRAFcap-M224 \\
65.2586 & 5.689 & ATCGA+GATTT+CTCTGTAG+CTA & BRAFcap-M929 \\
65.2567 & 5.687 & ATCGA+GATTTCT+CTGTAG+CTA & BRAFcap-M948 \\
65.2472 & 5.678 & ATCG+AGATTTCTC+TGTAG+CTA & BRAFcap-M835 \\
65.2339 & 5.665 & A+T+CGAGATTTCT+CTGTAGCTA & BRAFcap-M223 \\
65.232 & 5.663 & A+T+CGAGATTT+CTCTGTAGCTA & BRAFcap-M221 \\
\hline 60079 &
\end{tabular}

Continued on next page 
Table S5 - Continued from previous page

\begin{tabular}{|c|c|c|c|}
\hline$T_{m}$ & $\Delta T_{m}$ & Probe $\left(5^{\prime} \rightarrow 3^{\prime}\right)$ & ID \\
\hline 65.2263 & 5.657 & ATCG+AGATTT+CTCTGTAG+CTA & BRAFcap-M808 \\
\hline 65.2244 & 5.655 & ATCG+AGATTTCT+CTGTAG+CTA & BRAFcap-M827 \\
\hline 65.2244 & 5.655 & ATCGA+GATTTCTC+TGTAG+CTA & BRAFcap-M956 \\
\hline 65.1787 & 5.609 & ATCGAG+ATTTCTCTGTAG+ $+\mathbf{C}+\mathbf{T A}$ & BRAFcap-M1088 \\
\hline 65.1711 & 5.602 & AT+CGAGATTTCTC+TGTAGC+TA & BRAFcap-M545 \\
\hline 65.1635 & 5.594 & AT+CGAGA+TTTCTCTGTAGC+TA & BRAFcap-M482 \\
\hline 65.1635 & 5.594 & AT $+\mathbf{C G A G A T T T C T}+\mathbf{C}+\mathbf{T G T A G C T A}$ & BRAFcap-M531 \\
\hline 65.1559 & 5.587 & $\mathrm{AT}+\mathbf{C G A G A T T T}+\mathbf{C T C T G T A G C}+\mathbf{T A}$ & BRAFcap-M518 \\
\hline 65.154 & 5.585 & AT+CGAGATTTCT+CTGTAGC+TA & BRAFcap-M537 \\
\hline 65.1349 & 5.566 & 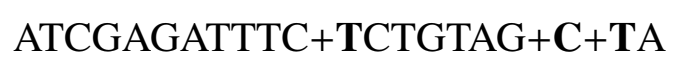 & BRAFcap-M1428 \\
\hline 65.133 & 5.564 & AT+CGAGATTTCTC+TGTA+GCTA & BRAFcap-M543 \\
\hline 64.9154 & 5.346 & ATCGAGAT+TTCT+CTGTAG+CTA & BRAFcap-M1225 \\
\hline 61.2724 & 1.703 & ATC+GAGATTTCTCTGTAGCT+A & BRAFcap-M728 \\
\hline 61.2047 & 1.635 & ATCGAGATT+TCTCT+GTAGCTA & BRAFcap-M1303 \\
\hline 61.1965 & 1.627 & +ATCGAGATT+TCTCTGTAGCT+A & BRAFcap-M145 \\
\hline 61.1636 & 1.594 & ATCGAGATTTCTCTG+TAGCTA & BRAFcap-M1521 \\
\hline 61.1471 & 1.578 & A+TCGAGATTTCTCTGTAGCT+A & BRAFcap-M402 \\
\hline 61.1451 & 1.576 & $+\mathbf{A}+\mathbf{T C G A G A T T T C T C T G T A G C T}+\mathbf{A}$ & BRAFcap-M21 \\
\hline 61.0854 & 1.516 & +ATCGAGATTTCTCTGT+AGCTA & BRAFcap-M197 \\
\hline 61.0565 & 1.487 & ATCGAG+ATTTCTCTGTAGCTA & BRAFcap-M987 \\
\hline 61.0132 & 1.444 & +ATCGAGATTTCTCT+GTAGCTA & BRAFcap-M184 \\
\hline 60.9926 & 1.423 & +ATCGAGAT+TTCTCTGTAGCTA & BRAFcap-M121 \\
\hline 60.9926 & 1.423 & ATCGAGA+TTTCTCTGTAGCTA & BRAFcap-M1093 \\
\hline 60.972 & 1.403 & ATCGAGATTTCTCTGT + AGCT $+\mathbf{A}$ & BRAFcap-M1547 \\
\hline 60.9141 & 1.345 & ATCGAGATTTC+TCTGTAGCTA & BRAFcap-M1387 \\
\hline 60.9017 & 1.332 & ATCGAGATTTCTCT+GTAGCT $+\mathbf{A}$ & BRAFcap-M1520 \\
\hline
\end{tabular}

Continued on next page

SI-12 
Table S5 - Continued from previous page

\begin{tabular}{l|l|l|l}
\hline$T_{m}$ & $\Delta T_{m}$ & Probe $\left(5^{\prime} \rightarrow 3^{\prime}\right)$ & ID \\
\hline 60.8893 & 1.320 & ATC+GAGATTTCTCTGTAGCTA & BRAFcap-M575 \\
60.8852 & 1.316 & ATCGAGAT+TTCTCTGTAGCT+A & BRAFcap-M1263 \\
60.7651 & 1.196 & A+TCGAGATTTCTCTGTAGCTA & BRAFcap-M212 \\
60.7631 & 1.194 & + +ATCGAGATT+TCTCTGTAGCTA & BRAFcap-M134 \\
60.7112 & 1.142 & + +A+TCGAGATTTCTCTGTAGCTA & BRAFcap-M2 \\
60.6531 & 1.084 & ATCGAGATT+TCTCTGTAGCT+A & BRAFcap-M1330 \\
60.597 & 1.028 & ATCGAGATTTCTCTGT+AGCTA & BRAFcap-M1537 \\
60.522 & 0.953 & +ATCGAGATTTCTCTGTAGCT+A & BRAFcap-M211 \\
60.522 & 0.953 & ATCGAGATTTCTCT+GTAGCTA & BRAFcap-M1499 \\
60.5033 & 0.934 & ATCGAGAT+TTCTCTGTAGCTA & BRAFcap-M1185 \\
60.2715 & 0.702 & ATCGAGATT+TCTCTGTAGCTA & BRAFcap-M1264 \\
60.0743 & 0.505 & +ATCGAGATTTCTCTGTAGCTA & BRAFcap-M1 \\
59.9627 & 0.393 & ATCGAGATTTCTCTGTAGCT+A & BRAFcap-M1561 \\
59.5693 & 0.000 & ATCGAGATTTCTCTGTAGCTA & BRAFcap-CTRL \\
\hline
\end{tabular}


Table S6: Predicted temperatures for BRAF linker LNA modified probe at medium salt concentration using the calculated parameters at high salt concentration from EE-DL minimization and applying salt correction for DNA[5] sequences. LNA modifications are preceded by a plus sign and marked in bold. A cut-off was made in the tables to show only the 30 sequences with the highest and lowest temperature difference between the modified and nonmodified probe, the total number of predicted sequences was 834. As comparative, we also show the $\Delta T_{m}$ of each modified sequence and its non-modified analog. The chosen probe is highlighted in yellow.

\begin{tabular}{l|l|l|l}
\hline$T_{m}$ & $\Delta T_{m}$ & Probe $\left(5^{\prime} \rightarrow 3^{\prime}\right)$ & ID \\
\hline 50.7521 & 0 & CAACTGTTCAAACTGAT & BRAFlin-CTRL \\
\hline 61.2632 & 10.511 & +CAA+CTGTTCAAA+CTGAT & BRAFlin-M42 \\
60.9684 & 10.216 & CAA+CTGTT+CAAAC+TGAT & BRAFlin-M417 \\
60.9594 & 10.207 & CAAC+TGTT+CAAA+CTGAT & BRAFlin-M495 \\
60.896 & 10.144 & +CAACTGTT+CAAA+CTGAT & BRAFlin-M97 \\
60.8749 & 10.123 & +CAA+CTGTT+CAAACTGAT & BRAFlin-M38 \\
60.7933 & 10.041 & CAA+CTG+TTCAAA+CTGAT & BRAFlin-M397 \\
60.754 & 10.002 & CAA+CTGTT+CAAA+CTGAT & BRAFlin-M416 \\
60.7268 & 9.975 & CAA+CTGTTCA+AA+CTGAT & BRAFlin-M431 \\
60.6239 & 9.872 & CAA+CTGTTCAAA+CTG+AT & BRAFlin-M445 \\
60.539 & 9.787 & CA+ACTGTT+CAAA+CTGAT & BRAFlin-M324 \\
60.5299 & 9.778 & CAA+CTGTT+CAA+ACTGAT & BRAFlin-M415 \\
60.4935 & 9.741 & CAA+CTGTT+CA+AACTGAT & BRAFlin-M414 \\
60.4722 & 9.720 & CAACTGTT+CA+AA+CTGAT & BRAFlin-M716 \\
60.4358 & 9.684 & CA+A+CTGTTCAAA+CTGAT & BRAFlin-M269 \\
60.4327 & 9.681 & CAA+CTGTTCAA+A+CTGAT & BRAFlin-M437 \\
60.3598 & 9.608 & CAA+C+TGTTCAAAC+TGAT & BRAFlin-M375 \\
60.3598 & 9.608 & CAAC+TGTTCAAA+C+TGAT & BRAFlin-M522 \\
60.3415 & 9.589 & CAA+CTGTTCAAA+CTGA+T & BRAFlin-M446 \\
60.3172 & 9.565 & CAA+CT+GTTCAAA+CTGAT & BRAFlin-M386 \\
60.2532 & 9.501 & CAA+CTGTT+CAAACTG+AT & BRAFlin-M419 \\
60.2319 & 9.480 & CAACTGTT+CAAA+CTG+AT & BRAFlin-M730 \\
\hline 60 & & & \\
\hline
\end{tabular}

Continued on next page 
Table S6 - Continued from previous page

\begin{tabular}{|c|c|c|c|}
\hline$T_{m}$ & $\Delta T_{m}$ & Probe $\left(5^{\prime} \rightarrow 3^{\prime}\right)$ & ID \\
\hline 60.2197 & 9.468 & $\mathbf{C}+\mathbf{A A}+\mathbf{C T G T T C A A A}+\mathbf{C T G A T}$ & BRAFlin-M163 \\
\hline 60.2197 & 9.468 & $\mathbf{C A A}+\mathbf{C T G T T C}+\mathbf{A A A}+\mathbf{C T G A T}$ & BRAFlin-M424 \\
\hline 60.1831 & 9.431 & $+\mathbf{C A A}+\mathbf{C T G T T C A A}+\mathbf{A C T G A T}$ & BRAFlin-M41 \\
\hline 60.1801 & 9.428 & $+\mathbf{C A}+\mathbf{A C T G T T C A A A}+\mathbf{C T G A T}$ & BRAFlin-M28 \\
\hline 60.174 & 9.422 & $\mathbf{C A}+\mathbf{A C}+\mathbf{T G T T C A A A}+\mathbf{C T G A T}$ & BRAFlin-M282 \\
\hline 60.174 & 9.422 & $\mathrm{CAA}+\mathbf{C T G T T C A A}+\mathbf{A C}+\mathbf{T G A T}$ & BRAFlin-M438 \\
\hline 53.9496 & 3.197 & CAAC+TGTTCAAACTGAT & BRAFlin-M457 \\
\hline 53.9462 & 3.194 & CAACTGTTCAAAC+TGAT & BRAFlin-M820 \\
\hline 53.926 & 3.174 & CAACT+GTTCAAACTGA+T & BRAFlin-M602 \\
\hline 53.926 & 3.174 & CAACTGT+TCA+AACTGAT & BRAFlin-M677 \\
\hline 53.9125 & 3.160 & +CAACTGTTCAAACTGAT & BRAFlin-M1 \\
\hline 53.8856 & 3.133 & CAACTGTTCAAACTG $+\mathbf{A}+\mathbf{T}$ & BRAFlin-M832 \\
\hline 53.8519 & 3.100 & $\mathbf{C}+\mathbf{A A C T G T T C A A A C T G A}+\mathbf{T}$ & BRAFlin-M258 \\
\hline 53.8451 & 3.093 & CAACTGTTC + AAACTGA $+\mathbf{T}$ & BRAFlin-M770 \\
\hline 53.808 & 3.056 & $\mathbf{C}+\mathbf{A A C T}+\mathbf{G T T C A A A C T G A T}$ & BRAFlin-M181 \\
\hline & 3.056 & CAACT+GTTC+AAACTGAT & BRAFlin-M567 \\
\hline 53.7709 & 3.019 & CAACTGT+TCAAACT+GAT & BRAFlin-M699 \\
\hline 53.7304 & 2.978 & $\mathbf{C}+\mathbf{A A C T G T T C}+\mathbf{A A A C T G A T}$ & BRAFlin-M223 \\
\hline 53.5139 & 2.762 & CAACTGT+TCAAACTGA+T & BRAFlin-M704 \\
\hline 53.4732 & 2.721 & CAACT+GT+TCAAACTGAT & BRAFlin-M548 \\
\hline 53.4189 & 2.667 & CA+ACTGTTCAAACTGAT & BRAFlin-M259 \\
\hline 53.4189 & 2.667 & CAACTGTTCAA+ACTGAT & BRAFlin-M793 \\
\hline 53.3985 & 2.646 & $\mathbf{C}+\mathbf{A A C T G T + T C A A A C T G A T ~}$ & BRAFlin-M204 \\
\hline 53.3951 & 2.643 & CAACTGT+TC+AAACTGAT & BRAFlin-M669 \\
\hline 53.2489 & 2.497 & CAACTGTTCAAACTG+AT & BRAFlin-M831 \\
\hline 52.88 & 2.128 & CAACTG+TTCAAACTGAT & BRAFlin-M603 \\
\hline
\end{tabular}

Continued on next page 


\begin{tabular}{l|l|l|l}
\multicolumn{6}{l}{ Table S6 - Continued from previous page } \\
\hline$T_{m}$ & $\Delta T_{m}$ & Probe (5' $\left.\rightarrow 3^{\prime}\right)$ & ID \\
\hline 52.8149 & 2.063 & CAACTGTTCA+AACTGAT & BRAFlin-M771 \\
52.6432 & 1.891 & CAACTGTTCAAACT+GAT & BRAFlin-M827 \\
52.3846 & 1.633 & CAACTGTTCAAACTGA+T & BRAFlin-M833 \\
52.3431 & 1.591 & CAACT+GTTCAAACTGAT & BRAFlin-M536 \\
52.267 & 1.515 & CAACTGTTC+AAACTGAT & BRAFlin-M742 \\
52.2636 & 1.511 & C+AACTGTTCAAACTGAT & BRAFlin-M138 \\
51.9233 & 1.171 & CAACTGT+TCAAACTGAT & BRAFlin-M659 \\
50.7521 & 0.000 & CAACTGTTCAAACTGAT & BRAFlin-CTRL \\
\hline
\end{tabular}


Table S7: Predicted temperatures for EGFR capture LNA modified probe at medium salt concentration using the calculated parameters at high salt concentration from EE-DL minimization and applying salt correction for DNA[5] sequences. LNA modifications are preceded by a plus sign and marked in bold. A cut-off was made in the tables to show only the 30 sequences with the highest and lowest temperature difference between the modified and nonmodified probe, the total number of predicted sequences was 2325. As comparative, we also show the $\Delta T_{m}$ of each modified sequence and its non-modified analog. The chosen probe is highlighted in yellow.

\begin{tabular}{|c|c|c|c|}
\hline$T_{m}$ & $\Delta T_{m}$ & Probe $\left(5^{\prime} \rightarrow 3^{\prime}\right)$ & ID \\
\hline 57.4035 & 0 & GAGAAAAAGTTTCTCATGTACAGT & EGFRcap-CTRL \\
\hline 62.3721 & 4.969 & GAGAAAAAGTTT+CT+CATGTA+CAGT & EGFRcap-M2045 \\
\hline 62.3707 & 4.967 & G+AGAAAAAGTTTCT+CATGTA+CAGT & EGFRcap-M483 \\
\hline 62.2985 & 4.895 & GA+GAAAAAGTTTCT+CATGTA+CAGT & EGFRcap-M715 \\
\hline 62.2958 & 4.892 & GAGAAAAAGTTTCT+CA+TGTA+CAGT & EGFRcap-M2164 \\
\hline 62.2006 & 4.797 & GAGAAAAA+GTTTCT+CATGTA+CAGT & EGFRcap-M1701 \\
\hline 62.1858 & 4.782 & GAGAAAAAGTTTCT+CATGTA+CA+GT & EGFRcap-M2188 \\
\hline 62.1764 & 4.773 & GAGAAAA+AGTTTCT+CATGTA+CAGT & EGFRcap-M1580 \\
\hline 62.1656 & 4.762 & GAGAAAAAG+TTTCT+CATGTA+CAGT & EGFRcap-M1807 \\
\hline 62.1629 & 4.759 & GAGAAAAAGTTTCT+CATG+TA+CAGT & EGFRcap-M2177 \\
\hline 62.1455 & 4.742 & GAGA+AAAAGTTTCT+CATGTA+CAGT & EGFRcap-M1117 \\
\hline 62.1441 & 4.741 & GAGAA+AAAGTTTCT+CATGTA+CAGT & EGFRcap-M1289 \\
\hline 62.1428 & 4.739 & GAGAAA+AAGTTTCT+CATGTA+CAGT & EGFRcap-M1443 \\
\hline 62.1172 & 4.714 & G+AGAAAAAGTTT+CTCATGTA+CAGT & EGFRcap-M462 \\
\hline 62.1118 & 4.708 & GAGAAAAAGTTTC $+\mathbf{T}+\mathbf{C A T G T A}+\mathbf{C A G T}$ & EGFRcap-M2101 \\
\hline 62.0862 & 4.683 & GAG+AAAAAGTTTCT+CATGTA+CAGT & EGFRcap-M926 \\
\hline 62.0822 & 4.679 & GA+GAAAAAGTTT+CTCATGTA+CAGT & EGFRcap-M694 \\
\hline 62.0607 & 4.657 & GAGAAAAAGTTTCT $+\mathbf{C A T G T A}+\mathbf{C}+\mathbf{A G T}$ & EGFRcap-M2187 \\
\hline 62.0539 & 4.650 & GAGAAAAAGTTT+CTCA+TGTA+CAGT & EGFRcap-M2062 \\
\hline 62.0054 & 4.602 & G+AGAAAAAGTTTCTCA+TGTA+CAGT & EGFRcap-M500 \\
\hline 61.9905 & 4.587 & GA+GAAAAAGTTTCTCA+TGTA+CAGT & EGFRcap-M732 \\
\hline 61.9662 & 4.563 & GAGAAAAA+GTTT+CTCATGTA+CAGT & EGFRcap-M1680 \\
\hline
\end{tabular}

Continued on next page 
Table S7 - Continued from previous page

\begin{tabular}{|c|c|c|c|}
\hline$T_{m}$ & $\Delta T_{m}$ & Probe $\left(5^{\prime} \rightarrow 3^{\prime}\right)$ & ID \\
\hline 61.9554 & 4.552 & GAGAAAAAGTTTCT+CAT+GTA+CAGT & EGFRcap-M2171 \\
\hline 61.9527 & 4.549 & GAGAAAAAGTTT+CTCATGTA $+\mathbf{C A}+\mathbf{G T}$ & EGFRcap-M2086 \\
\hline 61.9446 & 4.541 & GAGAAAA+AGTTT+CTCATGTA+CAGT & EGFRcap-M1559 \\
\hline 61.9405 & 4.537 & GAGAAAAAGT+TTCT+CATGTA+CAGT & EGFRcap-M1899 \\
\hline 61.9405 & 4.537 & GAGAAAAAGTTT+CTCATG+TA+CAGT & EGFRcap-M2075 \\
\hline 61.9324 & 4.529 & G+AGAAAAAGTTTCTCATG+TA+CAGT & EGFRcap-M513 \\
\hline 61.7603 & 4.357 & GAG+AAAAAGTTTCTCA+TGTA+CAGT & EGFRcap-M943 \\
\hline 58.6744 & 1.271 & GAGAAAAAGTTTCTCA+TGTACAGT & EGFRcap-M2233 \\
\hline 58.6478 & 1.244 & GAGAAAAAGTT+TCTCATGT+ACAGT & EGFRcap-M2012 \\
\hline 58.6227 & 1.219 & GAGAAAAAGTTTCTCAT+GTACAG+T & EGFRcap-M2283 \\
\hline 58.6183 & 1.215 & GAGAAAAAGT+TTCTCATGTACAG+T & EGFRcap-M1947 \\
\hline 58.6139 & 1.210 & +GAGAAAAAGTT+TCTCATGTACAGT & EGFRcap-M187 \\
\hline 58.6109 & 1.207 & GAGAAAAAGTTTCTC + ATGTACAG $+\mathbf{T}$ & EGFRcap-M2232 \\
\hline 58.5858 & 1.182 & GAGAAAAAGTT+TCTCAT+GTACAGT & EGFRcap-M1999 \\
\hline 58.5725 & 1.169 & GAGAAAAAGTT+TCTC+ATGTACAGT & EGFRcap-M1982 \\
\hline 58.5592 & 1.156 & GAGAAAAAGTTTCTCATG+TACAGT & EGFRcap-M2284 \\
\hline 58.5444 & 1.141 & GAGAAAAA+GTTTCTCATGTACAGT & EGFRcap-M1629 \\
\hline 58.5415 & 1.138 & GAGAAAAAGTTTCTCATGTACA+GT & EGFRcap-M2322 \\
\hline 58.537 & 1.133 & GAGAAAA+AGTTTCTCATGTACAGT & EGFRcap-M1492 \\
\hline 58.463 & 1.059 & GAG+AAAAAGTTTCTCATGTACAGT & EGFRcap-M764 \\
\hline 58.4481 & 1.045 & GAGAAAAAGTT+TCTCATGTACAG+T & EGFRcap-M2026 \\
\hline 58.3798 & 0.976 & GAGAAAAAGTTTC+TCATGTACAGT & EGFRcap-M2094 \\
\hline 58.3144 & 0.911 & GAGAAAAAG+TTTCTCATGTACAGT & EGFRcap-M1750 \\
\hline 58.3099 & 0.906 & GAGAAAAAGTTTCTCATGTAC+AGT & EGFRcap-M2318 \\
\hline 58.2816 & 0.878 & GAGAAA+AAGTTTCTCATGTACAGT & EGFRcap-M1338 \\
\hline 58.2801 & 0.877 & GAGA+AAAAGTTTCTCATGTACAGT & EGFRcap-M975 \\
\hline
\end{tabular}

Continued on next page 
Table S7 - Continued from previous page

\begin{tabular}{l|l|l|l}
\hline$T_{m}$ & $\Delta T_{m}$ & Probe $\left(5^{\prime} \rightarrow 3^{\prime}\right)$ & ID \\
\hline 58.2801 & 0.877 & GAGAA+AAAGTTTCTCATGTACAGT & EGFRcap-M1166 \\
58.1547 & 0.751 & GAGAAAAAGTTTCTCATGT+ACAGT & EGFRcap-M2300 \\
58.1158 & 0.712 & +GAGAAAAAGTTTCTCATGTACAGT & EGFRcap-M1 \\
58.0889 & 0.685 & GAGAAAAAGTTTCTCAT+GTACAGT & EGFRcap-M2262 \\
58.0829 & 0.679 & GAGAAAAAGT+TTCTCATGTACAGT & EGFRcap-M1856 \\
58.0784 & 0.675 & GAGAAAAAGTTTCTC+ATGTACAGT & EGFRcap-M2196 \\
57.9508 & 0.547 & GAGAAAAAGTTTCTCATGTACAG+T & EGFRcap-M2324 \\
57.9131 & 0.510 & GAGAAAAAGTT+TCTCATGTACAGT & EGFRcap-M1948 \\
57.4035 & 0.000 & GAGAAAAAGTTTCTCATGTACAGT & EGFRcap-CTRL \\
\hline
\end{tabular}


Table S8: Predicted temperatures for EGFR linker LNA modified probe at medium salt concentration using the calculated parameters at high salt concentration from EE-DL minimization and applying salt correction for DNA[5] sequences. LNA modifications are preceded by a plus sign and marked in bold. A cut-off was made in the tables to show only the 30 sequences with the highest and lowest temperature difference between the modified and nonmodified probe, the total number of predicted sequences was 988. As comparative, we also show the $\Delta T_{m}$ of each modified sequence and its non-modified analog. The chosen probe is highlighted in yellow.

\begin{tabular}{l|l|l|l}
\hline$T_{m}$ & $\Delta T_{m}$ & Probe $\left(5^{\prime} \rightarrow 3^{\prime}\right)$ & ID \\
\hline 54.4472 & 0 & TTGTTGGATCATATTCGT & EGFRlin-CTRL \\
\hline 62.3575 & 7.910 & TTGTTGGA+T+CA+TATTCGT & EGFRlin-M816 \\
62.2444 & 7.797 & TTGTTGGAT+CATA+TT+CGT & EGFRlin-M883 \\
62.1364 & 7.689 & TTGTTGGATCA+TA+TT+CGT & EGFRlin-M934 \\
62.131 & 7.684 & TTGTTGGAT+CA+TA+TTCGT & EGFRlin-M870 \\
62.1013 & 7.654 & TTGTTG+GA+T+CATATTCGT & EGFRlin-M703 \\
62.0635 & 7.616 & TTGTTG+GAT+CA+TATTCGT & EGFRlin-M714 \\
62.0554 & 7.608 & TTGTTGG+AT+CATATT+CGT & EGFRlin-M774 \\
62.0472 & 7.600 & TTGTTGG+AT+CA+TATTCGT & EGFRlin-M770 \\
61.9606 & 7.513 & TTG+TTGGAT+CA+TATTCGT & EGFRlin-M476 \\
61.9254 & 7.478 & TTG+TTGGATCA+TATT+CGT & EGFRlin-M495 \\
61.882 & 7.435 & TTGTT+GGAT+CATATT+CGT & EGFRlin-M651 \\
61.8495 & 7.402 & TTGTTGGA+T+CATA+TTCGT & EGFRlin-M818 \\
61.8332 & 7.386 & TTG+TTGGAT+CATATT+CGT & EGFRlin-M480 \\
61.8115 & 7.364 & TTGTTGGA+TCA+TATT+CGT & EGFRlin-M835 \\
61.7925 & 7.345 & TTGTTG+GATCA+TATT+CGT & EGFRlin-M733 \\
61.7843 & 7.337 & T+TGTTGGAT+CA+TATTCGT & EGFRlin-M249 \\
61.7653 & 7.318 & TTGT+TGGAT+CA+TATTCGT & EGFRlin-M568 \\
61.7545 & 7.307 & TTGTTGGAT+CATAT+T+CGT & EGFRlin-M887 \\
61.7164 & 7.269 & T+TGTTGGAT+CATATT+CGT & EGFRlin-M253 \\
61.7083 & 7.261 & TTGT+TGGAT+CATATT+CGT & EGFRlin-M572 \\
\hline 60.7083 & 7.261 & TTGTTGG+ATCATA+TT+CGT & EGFRlin-M800 \\
\hline 6 & & &
\end{tabular}

Continued on next page 
Table S8 - Continued from previous page

\begin{tabular}{|c|c|c|c|}
\hline$T_{m}$ & $\Delta T_{m}$ & Probe $\left(5^{\prime} \rightarrow 3^{\prime}\right)$ & ID \\
\hline 61.6511 & 7.204 & TTGTTGGAT+CA+TATTC+GT & EGFRlin-M873 \\
\hline 61.6321 & 7.185 & TTG+T+TGGAT+CATATTCGT & EGFRlin-M419 \\
\hline 61.5694 & 7.122 & TTGTT+GGATCA+TATT+CGT & EGFRlin-M666 \\
\hline 61.5667 & 7.119 & TTGTTGG+AT+CATA+TTCGT & EGFRlin-M772 \\
\hline 61.5422 & 7.095 & TTGTTGG+ATCA+TATT+CGT & EGFRlin-M789 \\
\hline 61.5176 & 7.070 & TTG+TTGGAT+CATA+TTCGT & EGFRlin-M478 \\
\hline 56.8219 & 2.375 & TT+GTTGGATCAT+ATTCGT & EGFRlin-M392 \\
\hline 56.816 & 2.369 & TT+GTTGGATCATATTCG+T & EGFRlin-M412 \\
\hline 56.8072 & 2.360 & TT+GTTGGATCATAT+TCGT & EGFRlin-M403 \\
\hline 56.7866 & 2.339 & TTGTTGGATC+AT+ATTCGT & EGFRlin-M904 \\
\hline 56.7836 & 2.336 & TTGTT+GGATCATATTCGT & EGFRlin-M611 \\
\hline 56.7836 & 2.336 & TTGTTGGATC+ATATTCG+T & EGFRlin-M924 \\
\hline 56.7748 & 2.328 & TTGTTGGATC+ATAT+TCGT & EGFRlin-M915 \\
\hline 56.7335 & 2.286 & TTGTTGGATCAT+A+TTCGT & EGFRlin-M948 \\
\hline 56.6568 & 2.210 & +TTGTTGGATCATATTCG+T & EGFRlin-M154 \\
\hline 56.5917 & 2.145 & TTGTTGG+ATCATATTCGT & EGFRlin-M757 \\
\hline 56.5622 & 2.115 & +TTGTTGGATCAT+ATTCGT & EGFRlin-M134 \\
\hline 56.5474 & 2.100 & +TTGTTGGATCATAT+TCGT & EGFRlin-M145 \\
\hline 56.5266 & 2.079 & TTGTTGGATCATA+TTCGT & EGFRlin-M963 \\
\hline 56.4645 & 2.017 & TTGTTGGATCAT+ATTCG+T & EGFRlin-M962 \\
\hline 56.4556 & 2.008 & TTGTTGGATCAT+AT+TCGT & EGFRlin-M953 \\
\hline 56.4437 & 1.996 & TTGTTGGATCATAT+TCG+T & EGFRlin-M980 \\
\hline 56.2775 & 1.830 & T+TGTTGGATCATATTCGT & EGFRlin-M155 \\
\hline 56.2745 & 1.827 & TTGT+TGGATCATATTCGT & EGFRlin-M519 \\
\hline 56.2715 & 1.824 & TTG+TTGGATCATATTCGT & EGFRlin-M413 \\
\hline 56.1882 & 1.741 & TTGTTGGA+TCATATTCGT & EGFRlin-M813 \\
\hline
\end{tabular}

Continued on next page 
Table S8 - Continued from previous page

\begin{tabular}{l|l|l|l}
\hline$T_{m}$ & $\Delta T_{m}$ & Probe $\left(5^{\prime} \rightarrow 3^{\prime}\right)$ & ID \\
\hline 56.06 & 1.613 & TTGTTGGATCATATTC+GT & EGFRlin-M985 \\
55.8238 & 1.377 & TT+GTTGGATCATATTCGT & EGFRlin-M292 \\
55.7999 & 1.353 & TTGTTGGATC+ATATTCGT & EGFRlin-M896 \\
55.5626 & 1.115 & +TTGTTGGATCATATTCGT & EGFRlin-M1 \\
55.4752 & 1.028 & TTGTTGGATCAT+ATTCGT & EGFRlin-M947 \\
55.4662 & 1.019 & TTGTTGGATCATATTCG+T & EGFRlin-M987 \\
55.4572 & 1.010 & TTGTTGGATCATAT+TCGT & EGFRlin-M974 \\
54.4472 & 0.000 & TTGTTGGATCATATTCGT & EGFRlin-CTRL \\
\hline
\end{tabular}


Table S9: Predicted temperatures for KRAS12D capture LNA modified probe at medium salt concentration using the calculated parameters at high salt concentration from EE-DL minimization and applying salt correction for DNA[5] sequences. LNA modifications are preceded by a plus sign and marked in bold. A cut-off was made in the tables to show only the 30 sequences with the highest and lowest temperature difference between the modified and nonmodified probe, the total number of predicted sequences was 1351. As comparative, we also show the $\Delta T_{m}$ of each modified sequence and its non-modified analog. The chosen probe is highlighted in yellow.

\begin{tabular}{|c|c|c|c|}
\hline$T_{m}$ & $\Delta T_{m}$ & Probe $\left(5^{\prime} \rightarrow 3^{\prime}\right)$ & ID \\
\hline 64.0694 & 0 & GCACTCTTGCCTACCCAATC & KRAS12cap-CTRL \\
\hline 71.3693 & 7.300 & $\mathbf{G}+\mathbf{C A}+\mathbf{C T C T T G C C}+\mathbf{T A C C C A A T C}$ & KRAS12cap-M219 \\
\hline 70.7611 & 6.692 & $\mathbf{G}+\mathbf{C A}+\mathbf{C T}+\mathbf{C T T G C C T A C C C A A T C ~}$ & KRAS12cap-M213 \\
\hline 70.5106 & 6.441 & $\mathrm{GCA}+\mathbf{C T C}+\mathbf{T T G C C T A}+\mathbf{C C C A A T C}$ & KRAS12cap-M557 \\
\hline 70.4782 & 6.409 & $\mathbf{G}+\mathbf{C A}+\mathbf{C T C}+\mathbf{T T G C C T A C C C A A T C ~}$ & KRAS12cap-M214 \\
\hline 70.4741 & 6.405 & $\mathbf{G}+\mathbf{C A C T C T T G C C}+\mathbf{T A C C C A}+\mathbf{A T C}$ & KRAS12cap-M325 \\
\hline 70.4538 & 6.384 & $\mathbf{G}+\mathbf{C A C T C T T G C C}+\mathbf{T A}+\mathbf{C C C A A T C}$ & KRAS12cap-M321 \\
\hline 70.326 & 6.257 & $\mathbf{G}+\mathbf{C A}+\mathbf{C T C T T G C C T A C C C A + \mathbf { A T C }}$ & KRAS12cap-M225 \\
\hline 70.2813 & 6.212 & $\mathrm{GCA}+\mathbf{C T}+\mathbf{C T T G C C}+\mathbf{T A C C C A A T C}$ & KRAS12cap-M541 \\
\hline 70.2468 & 6.177 & $\mathbf{G C A}+\mathbf{C T C T T G C C T A}+\mathbf{C C C A A T}+\mathbf{C}$ & KRAS12cap-M633 \\
\hline 70.2081 & 6.139 & $\mathbf{G C A}+\mathbf{C T C}+\mathbf{T T G C C}+\mathbf{T A C C C A A T C}$ & KRAS12cap-M555 \\
\hline 70.1572 & 6.088 & $\mathbf{G}+\mathbf{C A C T C}+\mathbf{T T G C C T A C C C A A T}+\mathbf{C}$ & KRAS12cap-M272 \\
\hline 70.1532 & 6.084 & $\mathbf{G C A}+\mathbf{C T C}+\mathbf{T T G C C T A C C}+\mathbf{C A A T C}$ & KRAS12cap-M559 \\
\hline 70.1185 & 6.049 & $\mathrm{GCA}+\mathbf{C T C T T G C C T A}+\mathbf{C C C A}+\mathbf{A T C}$ & KRAS12cap-M631 \\
\hline 70.1165 & 6.047 & $\mathbf{G}+\mathbf{C A C T}+\mathbf{C T T G C C T A}+\mathbf{C C C A A T C}$ & KRAS12cap-M252 \\
\hline 70.1145 & 6.045 & $\mathbf{G}+\mathbf{C A}+\mathbf{C T C T T G C C T A C C C A A}+\mathbf{T C}$ & KRAS12cap-M226 \\
\hline 70.1022 & 6.033 & $\mathbf{G}+\mathbf{C A C T C}+\mathbf{T T G C C T A C C C A}+\mathbf{A T C}$ & KRAS12cap-M270 \\
\hline 70.088 & 6.019 & $\mathbf{G}+\mathbf{C A}+\mathbf{C T C T T G C}+\mathbf{C T A C C C A A T C}$ & KRAS12cap-M218 \\
\hline 70.0839 & 6.014 & $\mathbf{G}+\mathbf{C A}+\mathbf{C T C T T G C C T A C C C A A T}+\mathbf{C}$ & KRAS12cap-M227 \\
\hline 70.0819 & 6.013 & $\mathbf{G}+\mathbf{C A C T C T T G C C T A}+\mathbf{C C}+\mathbf{C A A T C}$ & KRAS12cap-M338 \\
\hline 70.0615 & 5.992 & $\mathbf{G}+\mathbf{C A C T C T T G}+\mathbf{C}+\mathbf{C T A C C C A A T C}$ & KRAS12cap-M299 \\
\hline 70.0615 & 5.992 & GCA $+\mathbf{C T C T}+\mathbf{T G C C T A}+\mathbf{C C C A A T C}$ & KRAS12cap-M570 \\
\hline
\end{tabular}

Continued on next page 
Table S9 - Continued from previous page

\begin{tabular}{|c|c|c|c|}
\hline$T_{m}$ & $\Delta T_{m}$ & Probe $\left(5^{\prime} \rightarrow 3^{\prime}\right)$ & ID \\
\hline 70.0594 & 5.990 & $\mathbf{G}+\mathbf{C A C T}+\mathbf{C T T G C C}+\mathbf{T A C C C A A T C}$ & KRAS12cap-M250 \\
\hline 70.0533 & 5.984 & $\mathrm{GCA}+\mathbf{C T}+\mathbf{C}+\mathbf{T T G C C T A C C C A A T C}$ & KRAS12cap-M536 \\
\hline 70.0472 & 5.978 & $\mathbf{G}+\mathbf{C A C}+\mathbf{T C}+\mathbf{T T G C C T A C C C A A T C ~}$ & KRAS12cap-M230 \\
\hline 70.0451 & 5.976 & $\mathrm{GCA}+\mathbf{C T C T T G C C}+\mathbf{T A C C C A A T}+\mathbf{C}$ & KRAS12cap-M618 \\
\hline 70.0349 & 5.965 & GCACTC $+\mathbf{T T G C C}+\mathbf{T A}+\mathbf{C C C A A T C}$ & KRAS12cap-M931 \\
\hline 70.0207 & 5.951 & $\mathrm{GCA}+\mathbf{C T C T T G C}+\mathbf{C}+\mathbf{T A C C C A A T C}$ & KRAS12cap-M601 \\
\hline 66.4677 & 2.398 & GCACTCTTGCCTACCC $+\mathbf{A A T}+\mathbf{C}$ & KRAS12cap-M1343 \\
\hline 66.433 & 2.364 & GCAC+TCTTGCCTACCC+AATC & KRAS12cap-M766 \\
\hline 66.433 & 2.364 & GCACTCTTGCCTACCC $+\mathbf{A}+\mathbf{A T C}$ & KRAS12cap-M1338 \\
\hline 66.4308 & 2.361 & GCACTCTTGCCT+ACCCAA+TC & KRAS12cap-M1285 \\
\hline 66.4265 & 2.357 & GCACTCTT+GCCTACCC+AATC & KRAS12cap-M1110 \\
\hline 66.42 & 2.351 & +GCACTCTTGCCTACCC+AATC & KRAS12cap-M182 \\
\hline 66.3528 & 2.283 & GC+ACTCTTGCCTACCC+AATC & KRAS12cap-M508 \\
\hline 66.3528 & 2.283 & GCACTCTTGCCTACC+CAATC & KRAS12cap-M1326 \\
\hline 66.3441 & 2.275 & GCACTCT+TGCCTACCC+AATC & KRAS12cap-M1043 \\
\hline 66.2812 & 2.212 & GCACTCTTGCCTACCC $+\mathbf{A A}+\mathbf{T C}$ & KRAS12cap-M1341 \\
\hline 66.1268 & 2.057 & GCACTCTTGCC+TACCCAATC & KRAS12cap-M1222 \\
\hline 66.1115 & 2.042 & GCACTCTTGCCT+ACCC $+\mathbf{A A T C}$ & KRAS12cap-M1278 \\
\hline 66.0788 & 2.009 & GCACTCTTGCCTACCCA+A+TC & KRAS12cap-M1345 \\
\hline 66.057 & 1.988 & GCACT+CTTGCCTACCCAATC & KRAS12cap-M776 \\
\hline 65.7641 & 1.695 & GCACTC+TTGCCTACCCAATC & KRAS12cap-M882 \\
\hline 65.729 & 1.660 & GCACTCTTGC+CTACCCAATC & KRAS12cap-M1176 \\
\hline 65.538 & 1.469 & GCACTCTTGCCTACCCAAT+C & KRAS12cap-M1350 \\
\hline 65.527 & 1.458 & GCACTCTT+GCCTACCCAATC & KRAS12cap-M1053 \\
\hline 65.516 & 1.447 & +GCACTCTTGCCТАCCCAАТC & KRAS12cap-M1 \\
\hline 65.5027 & 1.433 & GCAC+TCTTGCCTACCCAATC & KRAS12cap-M655 \\
\hline
\end{tabular}

Continued on next page 
Table S9 - Continued from previous page

\begin{tabular}{l|l|l|l}
\hline$T_{m}$ & $\Delta T_{m}$ & Probe $\left(5^{\prime} \rightarrow 3^{\prime}\right)$ & ID \\
\hline 65.4785 & 1.409 & GCACTCTTGCCTAC+CCAATC & KRAS12cap-M1310 \\
65.419 & 1.350 & GC+ACTCTTGCCTACCCAATC & KRAS12cap-M364 \\
65.4168 & 1.347 & GCACTCTTGCCTACCCA+ATC & KRAS12cap-M1344 \\
65.4146 & 1.345 & GCACTCT+TGCCTACCCAATC & KRAS12cap-M974 \\
65.3595 & 1.290 & GCACTCTTGCCTACCCAA+TC & KRAS12cap-M1348 \\
65.1804 & 1.111 & GCACTCTTGCCT+ACCCAATC & KRAS12cap-M1259 \\
65.0319 & 0.962 & GCACTCTTGCCTACCC+AATC & KRAS12cap-M1337 \\
64.0694 & 0.000 & GCACTCTTGCCTACCCAATC & KRAS12cap-CTRL \\
\hline
\end{tabular}


Table S10: Predicted temperatures for KRAS13D capture LNA modified probe at medium salt concentration using the calculated parameters at high salt concentration from EE-DL minimization and applying salt correction for DNA[5] sequences. LNA modifications are preceded by a plus sign and marked in bold. A cut-off was made in the tables to show only the 30 sequences with the highest and lowest temperature difference between the modified and nonmodified probe, the total number of predicted sequences was 1351. As comparative, we also show the $\Delta T_{m}$ of each modified sequence and its non-modified analog. The chosen probe is highlighted in yellow.

\begin{tabular}{|c|c|c|c|}
\hline$T_{m}$ & $\Delta T_{m}$ & Probe $\left(5^{\prime} \rightarrow 3^{\prime}\right)$ & ID \\
\hline 64.1551 & 0 & GCACTCTTGCCTACGCATTC & KRAS13cap-CTRL \\
\hline 70.8901 & 6.735 & GCA $+\mathbf{C T C T T G C C}+\mathbf{T A C G}+\mathbf{C A T T C}$ & KRAS13cap-M614 \\
\hline 70.8861 & 6.731 & $\mathbf{G}+\mathbf{C A}+\mathbf{C T C T T G C C}+\mathbf{T A C G C A T T C}$ & KRAS13cap-M219 \\
\hline 70.7793 & 6.624 & $\mathbf{G}+\mathbf{C A C T C T T G C C T A}+\mathbf{C G C A}+\mathbf{T T C}$ & KRAS13cap-M340 \\
\hline 70.7309 & 6.576 & GCACTCTTGCCTA $+\mathbf{C G}+\mathbf{C A}+\mathbf{T T C}$ & KRAS13cap-M1297 \\
\hline 70.7067 & 6.552 & GCA+CTCTTGCC+TA+CGCATTC & KRAS13cap-M612 \\
\hline 70.6663 & 6.511 & $\mathbf{G C A}+\mathbf{C T}+\mathbf{C T T G C C T A C G}+\mathbf{C A T T C}$ & KRAS13cap-M545 \\
\hline 70.6481 & 6.493 & $\mathbf{G}+\mathbf{C A}+\mathbf{C T}+\mathbf{C T T G C C T A C G C A T T C ~}$ & KRAS13cap-M213 \\
\hline 70.6158 & 6.461 & $\mathbf{G C A}+\mathbf{C T}+\mathbf{C T T G C C T A}+\mathbf{C G C A T T C}$ & KRAS13cap-M543 \\
\hline 70.5227 & 6.368 & GCACTCTTGCC $+\mathbf{T A}+\mathbf{C G C A}+\mathbf{T T C}$ & KRAS13cap-M1235 \\
\hline 70.464 & 6.309 & $\mathbf{G}+\mathbf{C A}+\mathbf{C T C T T G C C T A C G C A}+\mathbf{T T C}$ & KRAS13cap-M225 \\
\hline 70.4579 & 6.303 & GCACT+CTTGCCTA+CGCA+TTC & KRAS13cap-M858 \\
\hline 70.4518 & 6.297 & $\mathbf{G C A}+\mathbf{C T C T T G C C T A C G}+\mathbf{C A}+\mathbf{T T C}$ & KRAS13cap-M642 \\
\hline 70.3869 & 6.232 & $\mathbf{G}+\mathbf{C A C T C T T G C C T A C G}+\mathbf{C A T T}+\mathbf{C}$ & KRAS13cap-M353 \\
\hline 70.3727 & 6.218 & $\mathbf{G C A}+\mathbf{C T C T T G C C T A}+\mathbf{C G C A}+\mathbf{T T C}$ & KRAS13cap-M631 \\
\hline 70.3605 & 6.205 & $\mathbf{G}+\mathbf{C A C T C T T G C C T A}+\mathbf{C G C A T T}+\mathbf{C}$ & KRAS13cap-M342 \\
\hline 70.3342 & 6.179 & GCACTCTTGCCTA $+\mathbf{C G}+\mathbf{C A T T}+\mathbf{C}$ & KRAS13cap-M1299 \\
\hline 70.3281 & 6.173 & $\mathbf{G C A}+\mathbf{C T C}+\mathbf{T T G C C T A C G}+\mathbf{C A T T C}$ & KRAS13cap-M559 \\
\hline 70.3078 & 6.153 & $\mathbf{G}+\mathbf{C A}+\mathbf{C T C}+\mathbf{T T G C C T A C G C A T T C}$ & KRAS13cap-M214 \\
\hline 70.2996 & 6.144 & $\mathbf{G}+\mathbf{C A C T C}+\mathbf{T T G C C T A C G C A}+\mathbf{T T C}$ & KRAS13cap-M270 \\
\hline 70.2895 & 6.134 & $\mathrm{GCA}+\mathbf{C T}+\mathbf{C T T G C C}+\mathbf{T A C G C A T T C}$ & KRAS13cap-M541 \\
\hline 70.2854 & 6.130 & $\mathbf{G}+\mathbf{C A C}+\mathbf{T C T T G C C T A}+\mathbf{C G C A T T C}$ & KRAS13cap-M237 \\
\hline
\end{tabular}

Continued on next page 
Table S10 - Continued from previous page

\begin{tabular}{|c|c|c|c|}
\hline$T_{m}$ & $\Delta T_{m}$ & Probe $\left(5^{\prime} \rightarrow 3^{\prime}\right)$ & ID \\
\hline 70.2773 & 6.122 & $\mathbf{G}+\mathbf{C A C}+\mathbf{T C T T G C C T A C G}+\mathbf{C A T T C}$ & KRAS13cap-M239 \\
\hline 70.2712 & 6.116 & GCACTC+TTGCCTACG+CA+TTC & KRAS13cap-M961 \\
\hline 70.261 & 6.106 & $\mathrm{GCA}+\mathbf{C T C}+\mathbf{T T G C C}+\mathbf{T A C G C A T T C}$ & KRAS13cap-M555 \\
\hline 70.2468 & 6.092 & $\mathbf{G C A}+\mathbf{C T C}+\mathbf{T T G C C T A}+\mathbf{C G C A T T C}$ & KRAS13cap-M557 \\
\hline 70.2102 & 6.055 & GCA+CTCTTGCC+TACGCA+TTC & KRAS13cap-M616 \\
\hline 70.1959 & 6.041 & GCACTCT+TGCC $+\mathbf{T A}+\mathbf{C G C A T T C}$ & KRAS13cap-M1010 \\
\hline 68.8296 & 4.674 & GCACTCTTGCCTA+CGCA+TTC & KRAS13cap-M1304 \\
\hline 66.4698 & 2.315 & GC+ACTCTTGCCTACGC+ATTC & KRAS13cap-M508 \\
\hline 66.4612 & 2.306 & GCACTCT+TGCCTACGC+ATTC & KRAS13cap-M1043 \\
\hline 66.4503 & 2.295 & GCACTCTTGCCTAC+GCAT+TC & KRAS13cap-M1323 \\
\hline 66.3051 & 2.150 & GCAC+TCTTGCCTACGCAT+TC & KRAS13cap-M773 \\
\hline 66.2703 & 2.115 & GCACTCTT+GCCTACGCAT+TC & KRAS13cap-M1117 \\
\hline 66.2616 & 2.106 & +GCACTCTTGCCTACGCAT+TC & KRAS13cap-M189 \\
\hline 66.2268 & 2.072 & GCACTCTTGCCT+ACGC+ATTC & KRAS13cap-M1278 \\
\hline 66.2247 & 2.070 & GC+ACTCTTGCCTACGCAT+TC & KRAS13cap-M515 \\
\hline 66.2181 & 2.063 & GCACTCT+TGCCTACGCAT+TC & KRAS13cap-M1050 \\
\hline 66.2073 & 2.052 & GCACTCTTGCC+TACGCATTC & KRAS13cap-M1222 \\
\hline 66.142 & 1.987 & GCACT+CTTGCCTACGCATTC & KRAS13cap-M776 \\
\hline 66.0745 & 1.919 & GCACTCTTGCCTACGC+A+TTC & KRAS13cap-M1338 \\
\hline 65.9828 & 1.828 & GCACTCTTGCCT+ACGCAT+TC & KRAS13cap-M1285 \\
\hline 65.8714 & 1.716 & GCACTCTTGCCTACGC+AT+TC & KRAS13cap-M1341 \\
\hline 65.8451 & 1.690 & GCACTC+TTGCCTACGCATTC & KRAS13cap-M882 \\
\hline 65.8079 & 1.653 & GCACTCTTGC+CTACGCATTC & KRAS13cap-M1176 \\
\hline 65.6983 & 1.543 & GCACTCTTGCCTACGCA+TTC & KRAS13cap-M1344 \\
\hline 65.6764 & 1.521 & GCACTCTTGCCTAC+GCATTC & KRAS13cap-M1310 \\
\hline 65.6215 & 1.466 & GCACTCTTGCCTACGCATT+ $+\mathbf{C}$ & KRAS13cap-M1350 \\
\hline
\end{tabular}

Continued on next page 
Table S10 - Continued from previous page

\begin{tabular}{l|l|l|l}
\hline$T_{m}$ & $\Delta T_{m}$ & Probe $\left(5^{\prime} \rightarrow 3^{\prime}\right)$ & ID \\
\hline 65.5995 & 1.444 & +GCACTCTTGCCTACGCATTC & KRAS13cap-M1 \\
65.5863 & 1.431 & GCAC+TCTTGCCTACGCATTC & KRAS13cap-M655 \\
65.5027 & 1.348 & GC+ACTCTTGCCTACGCATTC & KRAS13cap-M364 \\
65.5005 & 1.345 & GCACTCT+TGCCTACGCATTC & KRAS13cap-M974 \\
65.4939 & 1.339 & GCACTCTT+GCCTACGCATTC & KRAS13cap-M1053 \\
65.2623 & 1.107 & GCACTCTTGCCT+ACGCATTC & KRAS13cap-M1259 \\
65.1517 & 0.997 & GCACTCTTGCCTACGC+ATTC & KRAS13cap-M1337 \\
64.8986 & 0.743 & GCACTCTTGCCTACGCAT+TC & KRAS13cap-M1348 \\
64.1551 & 0.000 & GCACTCTTGCCTACGCATTC & KRAS13cap-CTRL \\
\hline
\end{tabular}


Table S11: Predicted temperatures for KRAS linker LNA modified probe at medium salt concentration using the calculated parameters at high salt concentration from EE-DL minimization and applying salt correction for DNA[5] sequences. LNA modifications are preceded by a plus sign and marked in bold. A cut-off was made in the tables to show only the 30 sequences with the highest and lowest temperature difference between the modified and nonmodified probe, the total number of predicted sequences was 834. As comparative, we also show the $\Delta T_{m}$ of each modified sequence and its non-modified analog. The chosen probe is highlighted in yellow.

\begin{tabular}{|c|c|c|c|}
\hline$T_{m}$ & $\Delta T_{m}$ & Probe $\left(5^{\prime} \rightarrow 3^{\prime}\right)$ & ID \\
\hline 48.1502 & 0 & TGAAGTCACATTATATA & KRASlin-CTRL \\
\hline 58.8304 & 10.680 & TGAAGT+CA+CATTATA+TA & KRASlin-M621 \\
\hline 58.8152 & 10.665 & TGAAGT+CA+CATTA+TATA & KRASlin-M619 \\
\hline 58.544 & 10.394 & TGAAGTCA $+\mathbf{C A T T A}+\mathbf{T A}+\mathbf{T A}$ & KRASlin-M734 \\
\hline 58.3759 & 10.226 & TGAA+GT+CA+CATTATATA & KRASlin-M472 \\
\hline 58.3636 & 10.213 & TGAAG $+\mathbf{T}+\mathbf{C A}+\mathbf{C A T T A T A T A}$ & KRASlin-M539 \\
\hline 58.333 & 10.183 & $\mathrm{TGA}+\mathbf{A G T}+\mathbf{C A}+\mathbf{C A T T A T A T A}$ & KRASlin-M393 \\
\hline 58.2932 & 10.143 & TGAA+GTCA $+\mathbf{C A T T A T A}+\mathbf{T A}$ & KRASlin-M498 \\
\hline 58.2778 & 10.128 & TGAA $+\mathbf{G T C A}+\mathbf{C A T T A}+\mathbf{T A T A}$ & KRASlin-M496 \\
\hline 58.2594 & & TGA+AGTCA+CATTATA+TA & KRASlin-M419 \\
\hline 58.241 & 10.091 & TGA+AGTCA+CATTA+TATA & KRASlin-M417 \\
\hline 58.2226 & 10.072 & TGAAGTCA+CA+TTATA+TA & KRASlin-M719 \\
\hline 58.1981 & 10.048 & TGAAGTCA+CA+TTA+TATA & KRASlin-M717 \\
\hline 58.1705 & 10.020 & TGAAGT $+\mathbf{C A}+\mathbf{C A}+\mathbf{T T A T A T A}$ & KRASlin-M616 \\
\hline & & TGAAGTCA $+\mathbf{C A}+\mathbf{T}+\mathbf{T A T A T A}$ & KRASlin-M715 \\
\hline 58.0936 & 9.943 & TG+AAGTCA+CATTATA+TA & KRASlin-M327 \\
\hline 58.0813 & 9.931 & TG+AAGTCA $+\mathbf{C A T T A}+\mathbf{T A T A}$ & KRASlin-M325 \\
\hline 58.0383 & 9.888 & TGAAGTCA+CAT+TATA+TA & KRASlin-M725 \\
\hline 58.0167 & 9.867 & TGAAGTCA+CAT+TA+TATA & KRASlin-M723 \\
\hline 57.9551 & 9.805 & $\mathrm{TG}+\mathbf{A A G T}+\mathbf{C A}+\mathbf{C A T T A T A T A}$ & KRASlin-M301 \\
\hline 57.9181 & 9.768 & $\mathrm{TGA}+\mathbf{A}+\mathbf{G T C A}+\mathbf{C A T T A T A T A}$ & KRASlin-M370 \\
\hline 57.912 & 9.762 & TGA $+\mathbf{A G T C A}+\mathbf{C A}+\mathbf{T T A T A T A}$ & KRASlin-M414 \\
\hline
\end{tabular}

Continued on next page 
Table S11 - Continued from previous page

\begin{tabular}{|c|c|c|c|}
\hline$T_{m}$ & $\Delta T_{m}$ & Probe $\left(5^{\prime} \rightarrow 3^{\prime}\right)$ & ID \\
\hline 57.8781 & 9.728 & TG+A $+\mathbf{A G T C A}+\mathbf{C A T T A T A T A ~}$ & KRASlin-M265 \\
\hline 57.8565 & 9.706 & TGAAGT $+\mathbf{C A}+\mathbf{C A T T A T}+\mathbf{A T A}$ & KRASlin-M620 \\
\hline 57.8503 & 9.700 & TGAAGT $+\mathbf{C A}+\mathbf{C A T T}+\mathbf{A T A T A}$ & KRASlin-M618 \\
\hline 57.7484 & 9.598 & TGAAGT $+\mathbf{C A}+\mathbf{C A T}+\mathbf{T A T A T A}$ & KRASlin-M617 \\
\hline 57.7267 & 9.577 & TGA+AGTCA+CAT+TATATA & KRASlin-M415 \\
\hline 57.6989 & 9.549 & TGAA+GTCA+CA+TTATATA & KRASlin-M493 \\
\hline 51.1563 & 3.006 & TGAAGTCACATT $+\mathbf{A T}+\mathbf{A T}+\mathbf{A}$ & KRASlin-M816 \\
\hline 51.053 & 2.903 & TGAAG+TCACATT+ATATA & KRASlin-M588 \\
\hline 51.053 & 2.903 & TGAAG+TCACATTAT+ATA & KRASlin-M597 \\
\hline 51.0012 & 2.851 & TGAAGTCACAT+TATAT+A & KRASlin-M808 \\
\hline 50.9529 & 2.803 & TGAAGTC + ACATTATAT $+\mathbf{A}$ & KRASlin-M704 \\
\hline 50.908 & 2.758 & TGAAGTCAC $+\mathbf{A T T A T}+\mathbf{A T A}$ & KRASlin-M765 \\
\hline 50.9045 & 2.754 & TGAAGTCAC+ATT+ATATA & KRASlin-M756 \\
\hline 50.8319 & 2.682 & TGAA+GTCACATTATATA & KRASlin-M457 \\
\hline 50.8181 & 2.668 & TGA+AGTCACATTATATA & KRASlin-M365 \\
\hline 50.7765 & 2.626 & +TGAAGTCACATTATAT+A & KRASlin-M137 \\
\hline 50.7315 & 2.581 & T+GAAGTCACATTATAT+A & KRASlin-M258 \\
\hline 50.61 & 2.460 & TG+AAGTCACATTATATA & KRASlin-M259 \\
\hline 50.5996 & 2.449 & TGAAGTCACA+TTATATA & KRASlin-M771 \\
\hline 50.5474 & 2.397 & TGAAG+TCACATTATAT+A & KRASlin-M602 \\
\hline 50.5266 & 2.376 & TGAAGTCACATT $+\mathbf{A T}+\mathbf{A T A}$ & KRASlin-M814 \\
\hline 50.4012 & 2.251 & TGAAGTCAC + ATTATAT $+\mathbf{A}$ & KRASlin-M770 \\
\hline 50.3593 & 2.209 & TGAAGTCACAT+TATATA & KRASlin-M793 \\
\hline 50.307 & 2.157 & TGAAGTC+ACATTATATA & KRASlin-M659 \\
\hline 50.0725 & 1.922 & T+GAAGTCACATTATATA & KRASlin-M138 \\
\hline 50.0199 & 1.870 & TGAAGTCACATT $+\mathbf{A T A T}+\mathbf{A}$ & KRASlin-M819 \\
\hline
\end{tabular}

Continued on next page 
Table S11 - Continued from previous page

\begin{tabular}{l|l|l|l}
\hline$T_{m}$ & $\Delta T_{m}$ & Probe $\left(5^{\prime} \rightarrow 3^{\prime}\right)$ & ID \\
\hline 50.0164 & 1.866 & TGAAGTCACATTAT+AT+A & KRASlin-M830 \\
50.0093 & 1.859 & +TGAAGTCACATTATATA & KRASlin-M1 \\
49.8899 & 1.740 & TGAAG+TCACATTATATA & KRASlin-M536 \\
49.7455 & 1.595 & TGAAGTCAC+ATTATATA & KRASlin-M742 \\
49.3597 & 1.209 & TGAAGTCACATT+ATATA & KRASlin-M809 \\
49.3597 & 1.209 & TGAAGTCACATTAT+ATA & KRASlin-M827 \\
48.8353 & 0.685 & TGAAGTCACATTATAT+A & KRASlin-M833 \\
48.1502 & 0.000 & TGAAGTCACATTATATA & KRASlin-CTRL \\
\hline
\end{tabular}


Table S12: MALDI-MS of purified oligonucleotides and their respective purities. LNA modifications are preceded by a plus sign and marked in bold. The 'target-binding, measured probe, is marked in red.

\begin{tabular}{lllllc}
\hline \hline Kas code & Name & Sequences $\left(5^{\prime} \rightarrow 3^{\prime}\right)$ & MW [g/mol] & MS [m/z] & Purity [\%] \\
\hline KAS209 & BRAFcap & TTTTTTTTTCCCCCCCCATCGAGAT+TTCT+CTGTAG+CTA & 12457.1 & 12455.9 & 90.6 \\
KAS210 & EGFRcap & TTTTTTTTCCCCCCCCGAG+AAAAGTTTCTCA+TGTA+CAGT & 13431.7 & 13432.8 & 98.3 \\
KAS211 & KRAS12cap & TTTTTTTTTCCCCCCCCGCACTCTTGCCTACCCA+ATC & 11934.7 & 11933.8 & 99.6 \\
KAS212 & KRAS13cap & TTTTTTTTT CCCCCCCCCGCACTCTGCCTA+CGCA+TTC & 12007.8 & 12008.3 & 99.2 \\
KAS213 & BRAFlink & GA+TGG+GAATA+CCAGAC+CAC+CTGTTTTCAA+CTGTT+CAAA+CTGAT & 13493.9 & 13493.0 & 97.8 \\
KAS214 & EGFRlink & GA+TGG+GAATA+CCAGAC+CAC+CTGTTTTTGAAGT+CA+CA+TTATATA & 13518.9 & 13519.3 & 95.2 \\
KAS215 & KRASlink & GA+TGG+GAATA+CCAGAC+CAC+CTGTTTTTTG+TTGGAT+CATA+TTCGT & 13823.0 & 13822.4 & 98.3 \\
\hline \hline
\end{tabular}

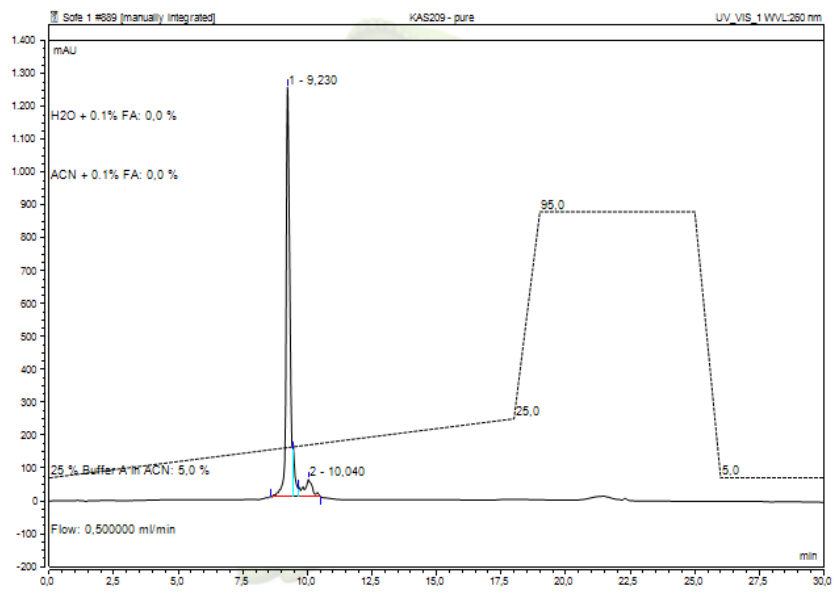

Figure S1: Representative UHPLC traces of $B R A F$ capture probe.

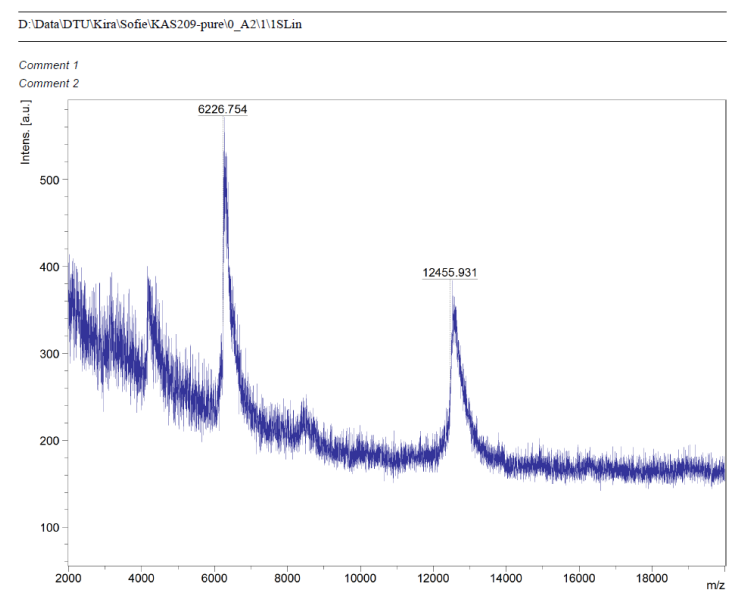

Figure S2: Representative MALDI-MS spectrum of $B R A F$ capture probe. 


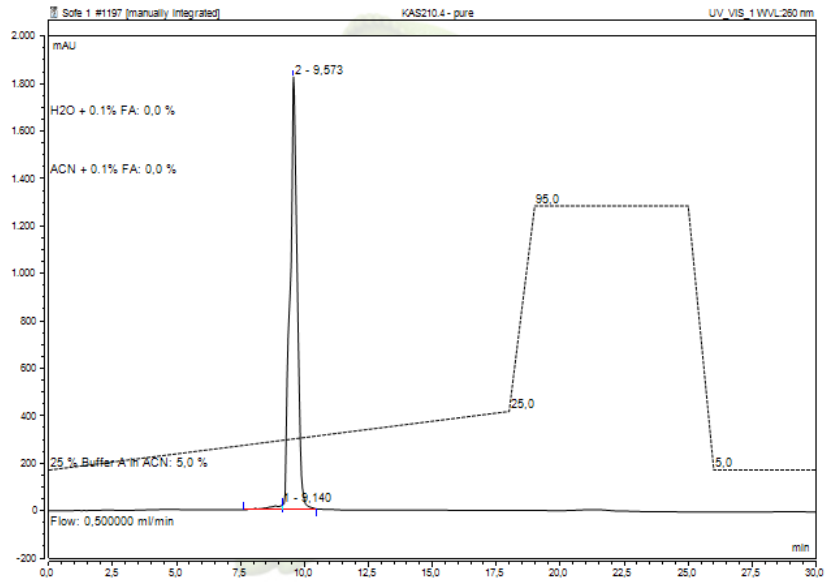

Figure S3: Representative UHPLC traces of EGFR capture probe.

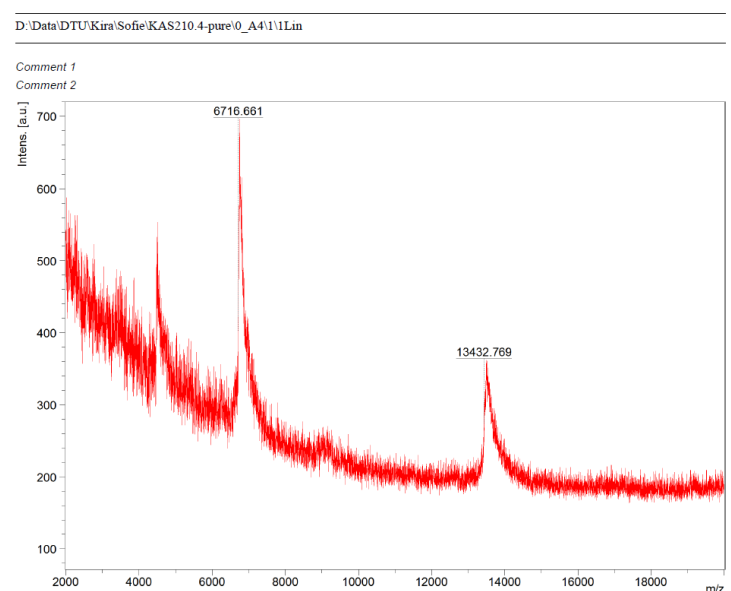

Figure S4: Representative MALDI-MS spectrum of EGFR capture probe.

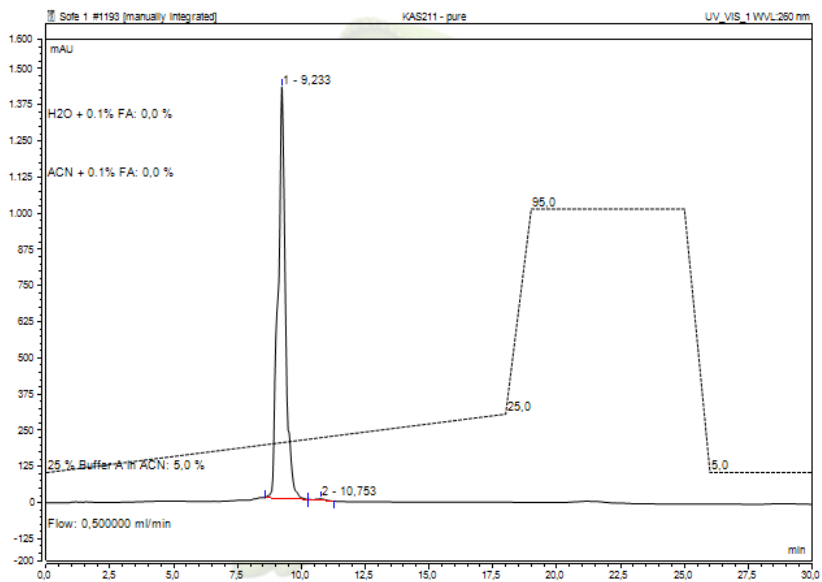

Figure S5: Representative UHPLC traces of KRAS12D capture probe. 


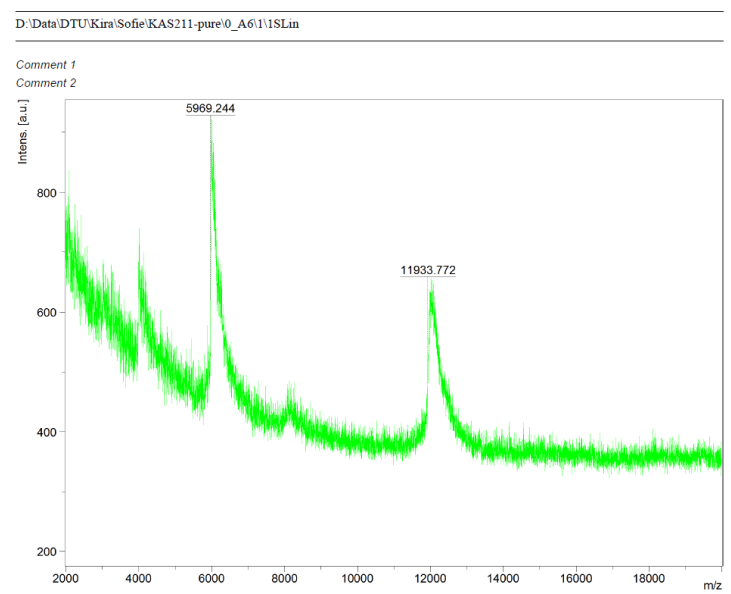

Figure S6: Representative MALDI-MS spectrum of KRAS12D capture probe.

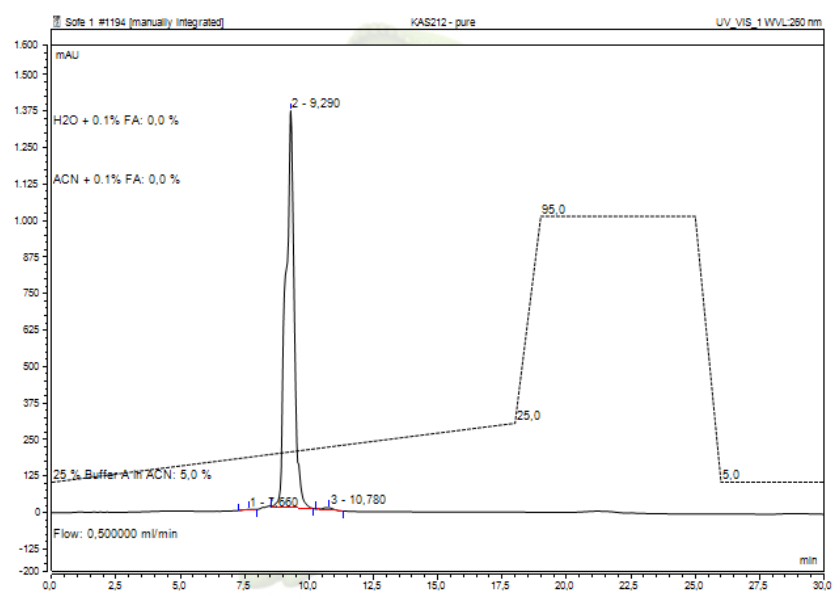

Figure S7: Representative UHPLC traces of KRAS13D capture probe.

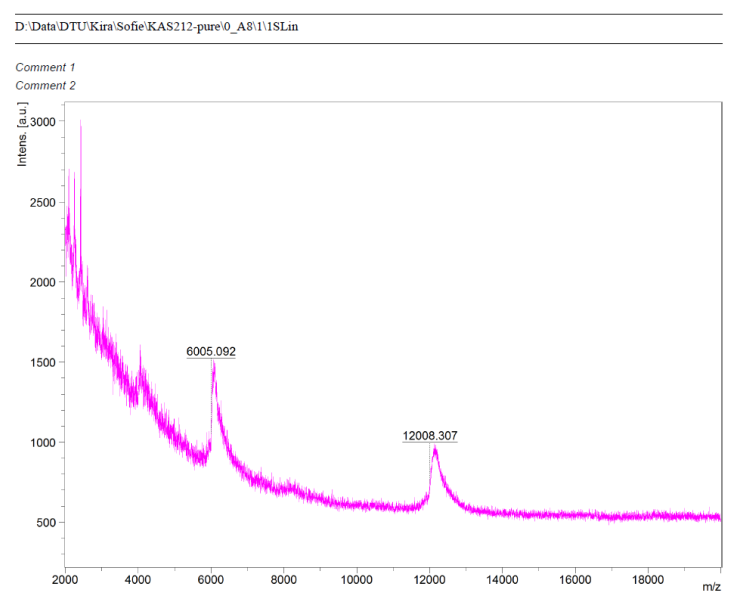

Figure S8: Representative MALDI-MS spectrum of KRAS13D capture probe. 


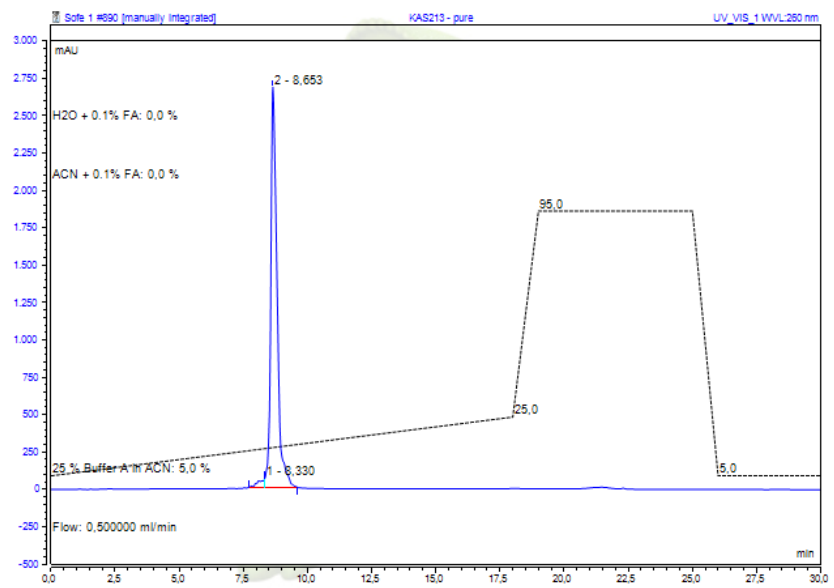

Figure S9: Representative UHPLC traces of $B R A F$ linker probe.

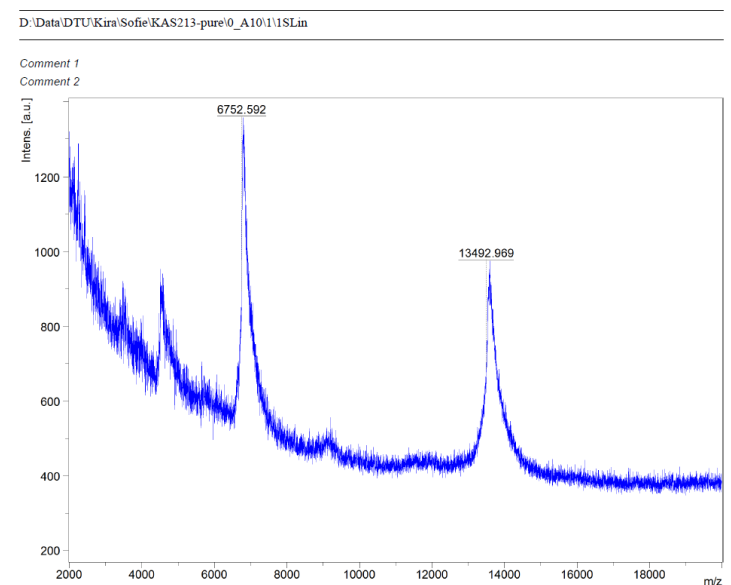

Figure S10: Representative MALDI-MS spectrum of $B R A F$ linker probe.

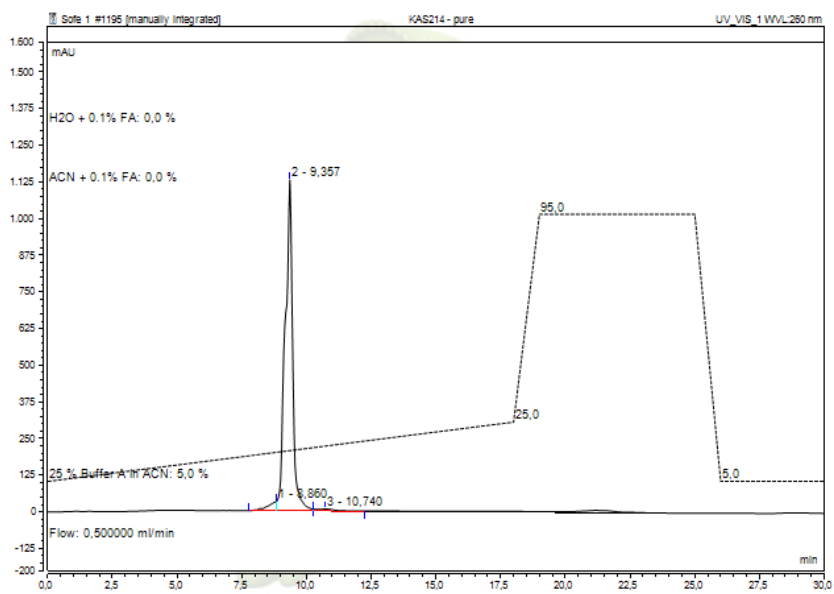

Figure S11: Representative UHPLC traces of EGFR linker probe. 


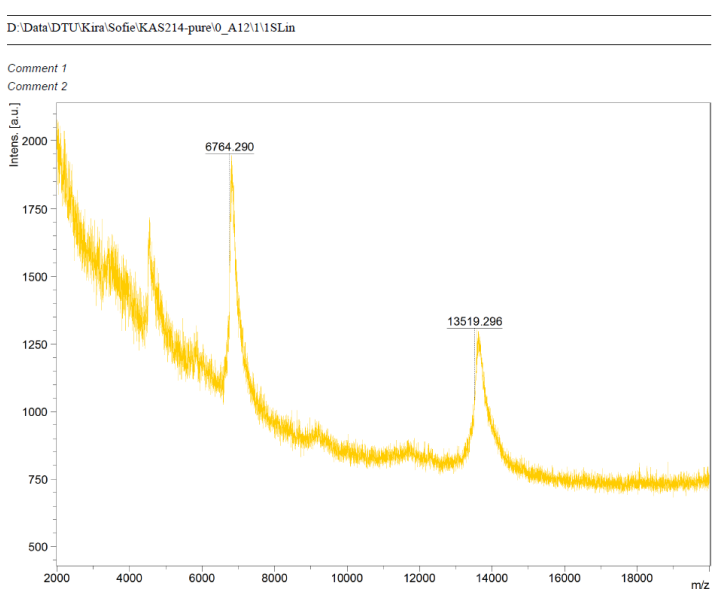

Figure S12: Representative MALDI-MS spectrum of EGFR linker probe.

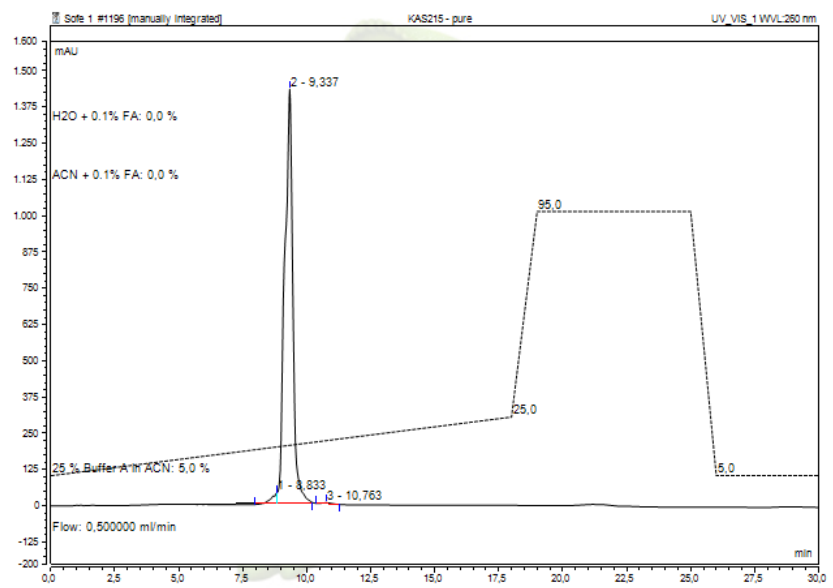

Figure S13: Representative UHPLC traces of KRAS linker probe.

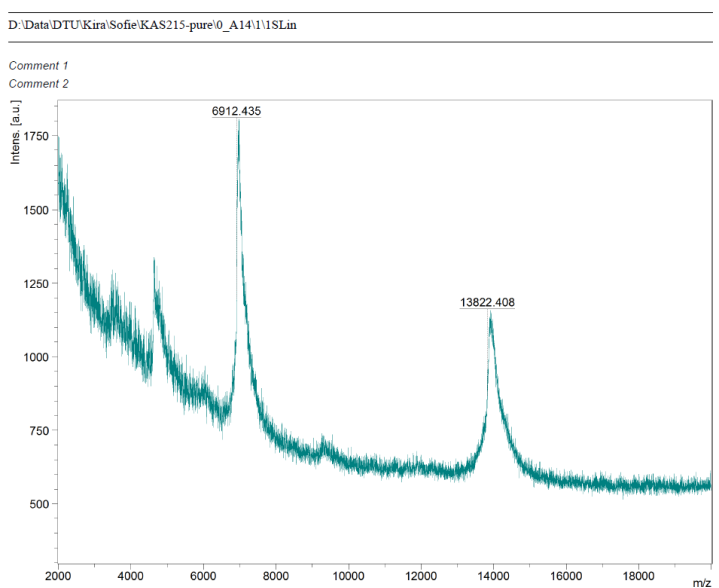

Figure S14: Representative MALDI-MS spectrum of $K R A S$ linker probe. 


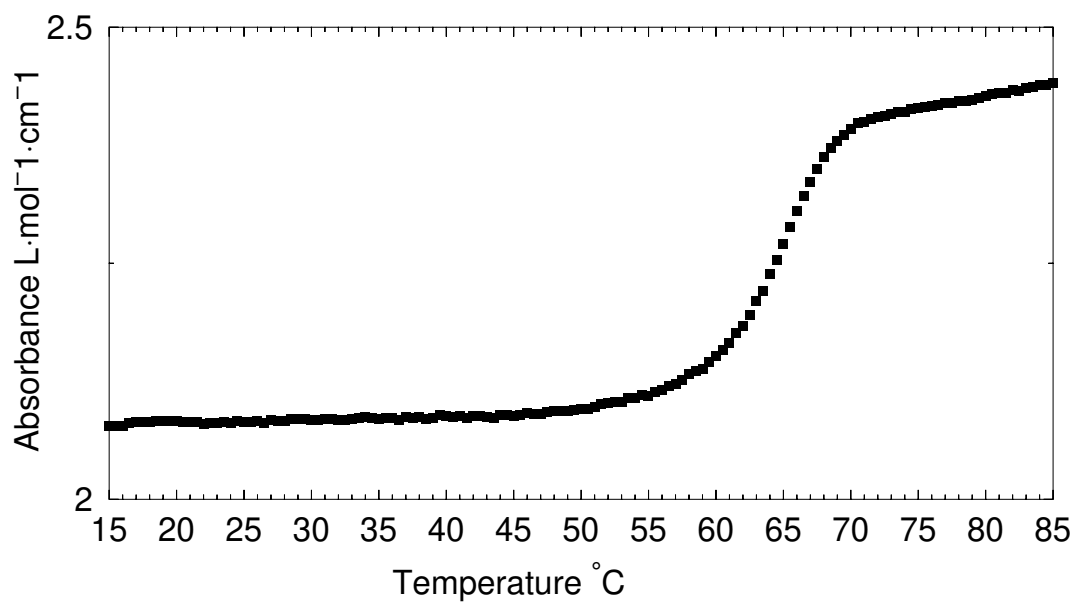

Figure S15: Representative $T_{m}$ curve of $B R A F$ capture probe.

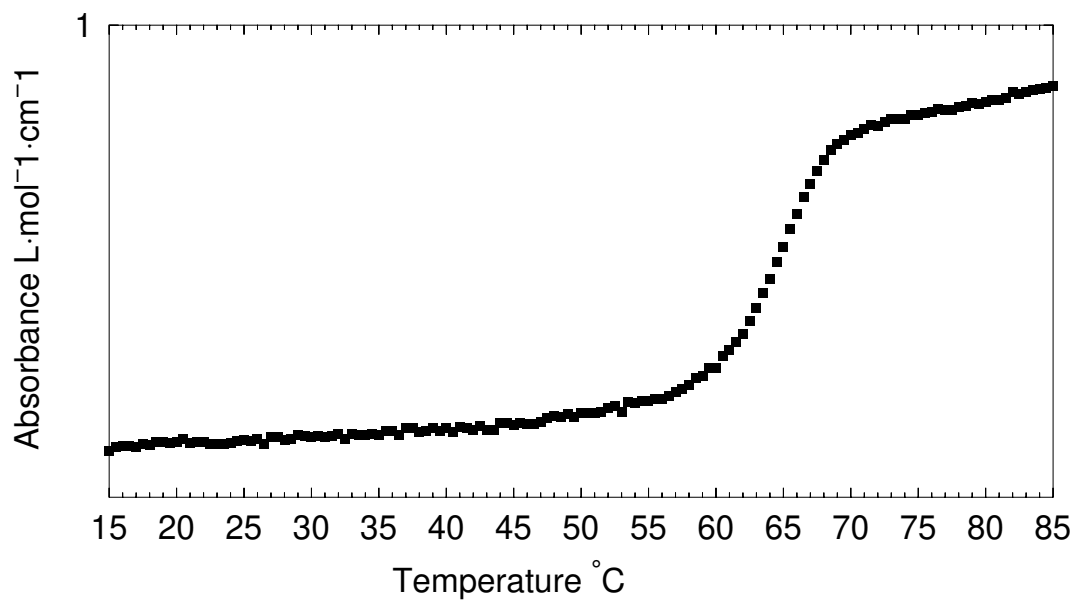

Figure S16: Representative $T_{m}$ curve of KRAS12D capture probe.

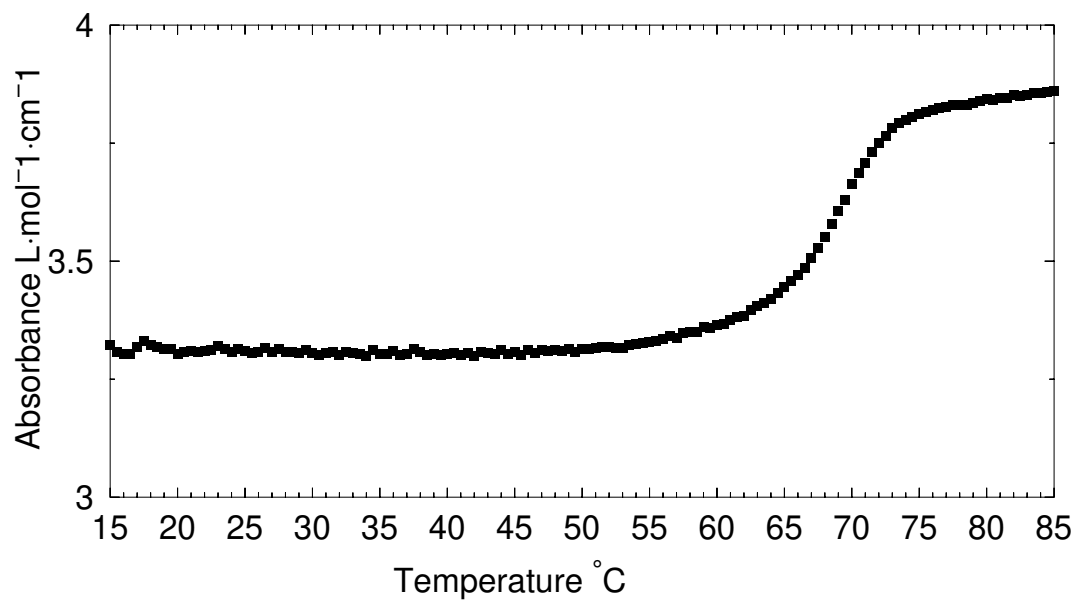

Figure S17: Representative $T_{m}$ curve of $K R A S 13 D$ capture probe. 


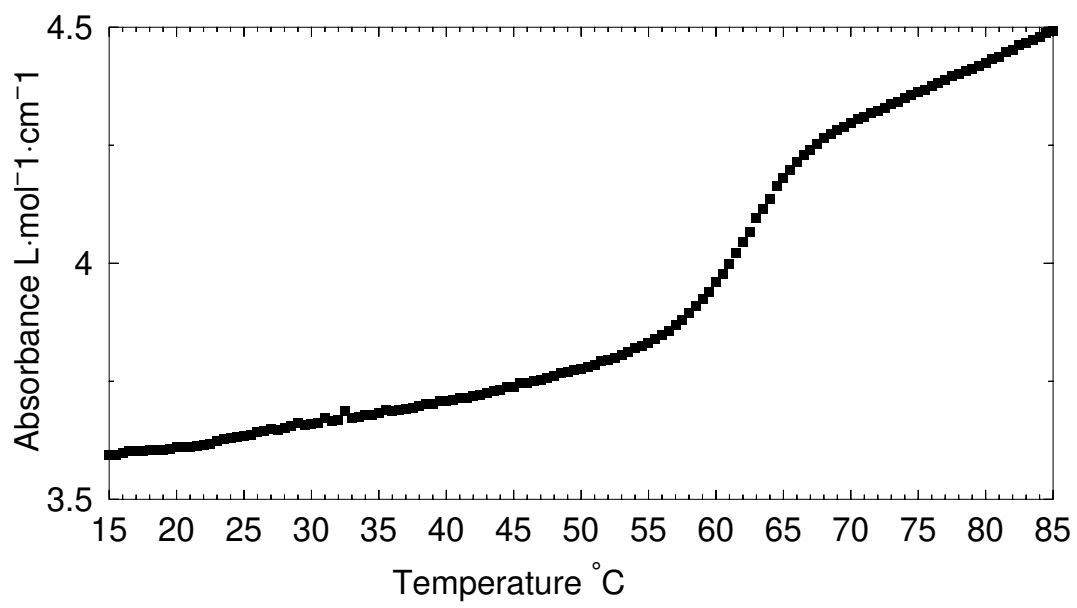

Figure S18: Representative $T_{m}$ curve of $B R A F$ linker probe.

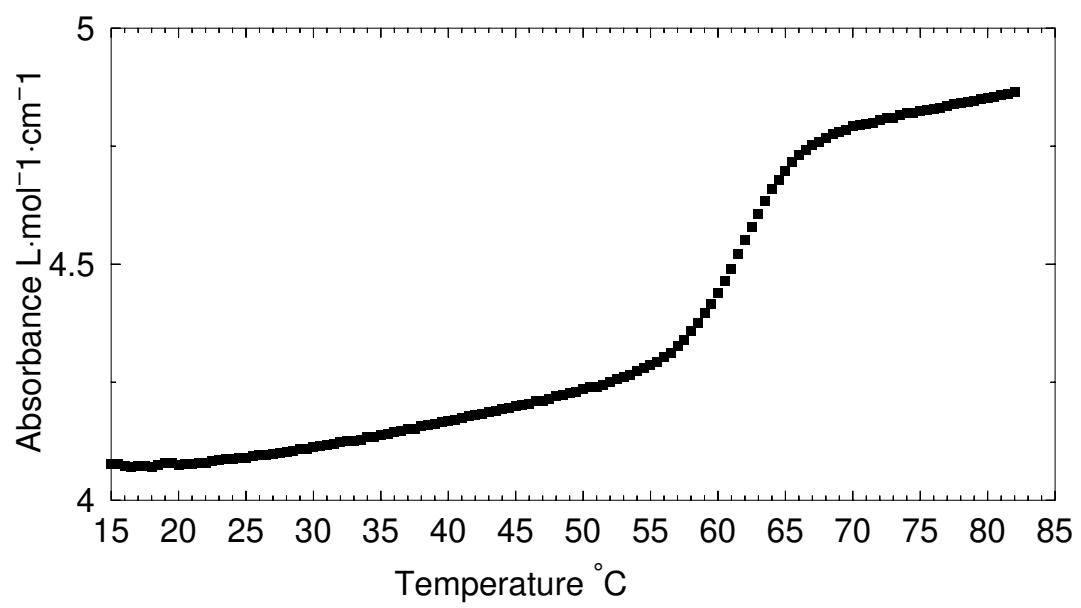

Figure S19: Representative $T_{m}$ curve of $E G F R$ linker probe.

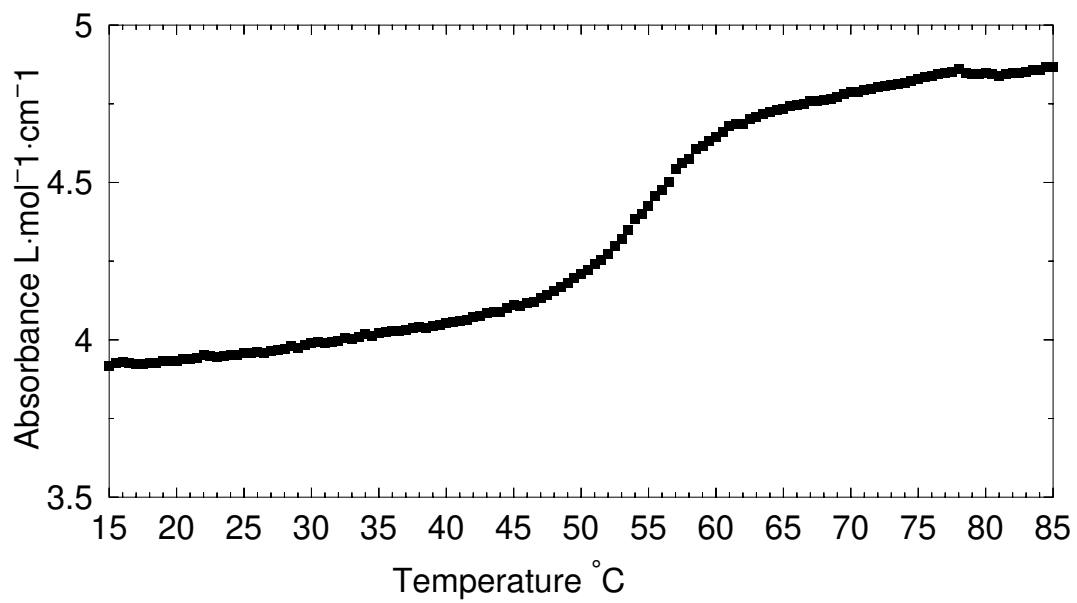

Figure S20: Representative $T_{m}$ curve of $K R A S$ linker probe. 


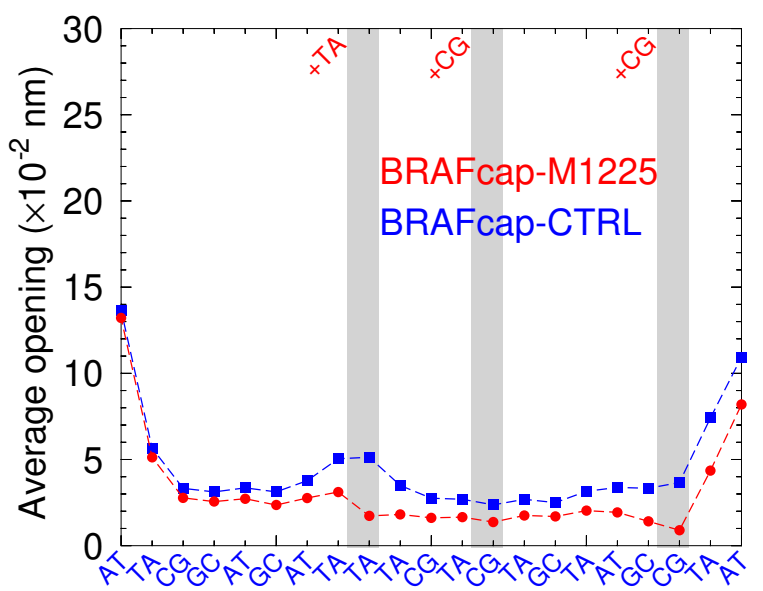

Figure S21: Average opening profile for $B R A F$ capture probe. Modified and non-modified probe are shown in red bullets and blue squares, respectively. LNA modifications are preceded by a plus sign and marked in bold. The location of the LNAs is indicated by the gray shaded area. Calculation was carried out at $220 \mathrm{~K}$ which has no relation to the melting temperature.

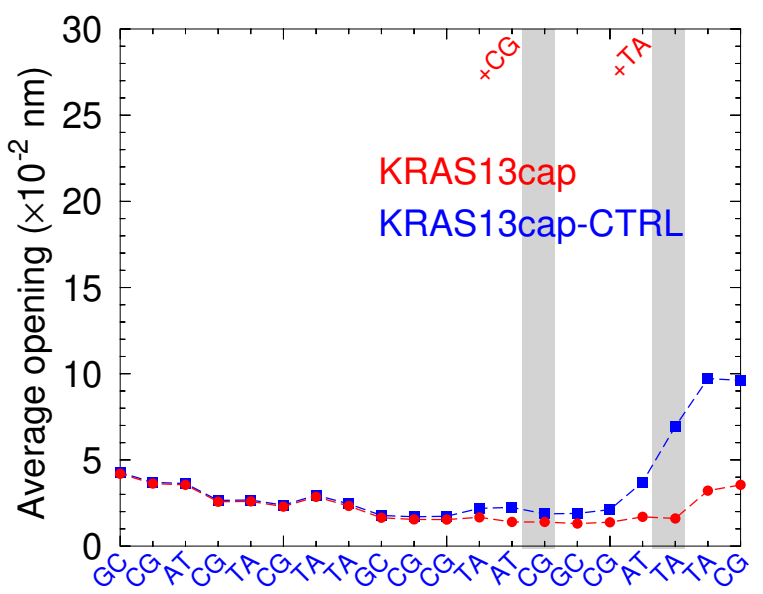

Figure S22: Average opening profile for KRAS13D capture probe. Modified and non-modified probe are shown in red bullets and blue squares, respectively. LNA modifications are preceded by a plus sign and marked in bold. The location of the LNAs is indicated by the gray shaded area. Calculation was carried out at $220 \mathrm{~K}$ which has no relation to the melting temperature. 


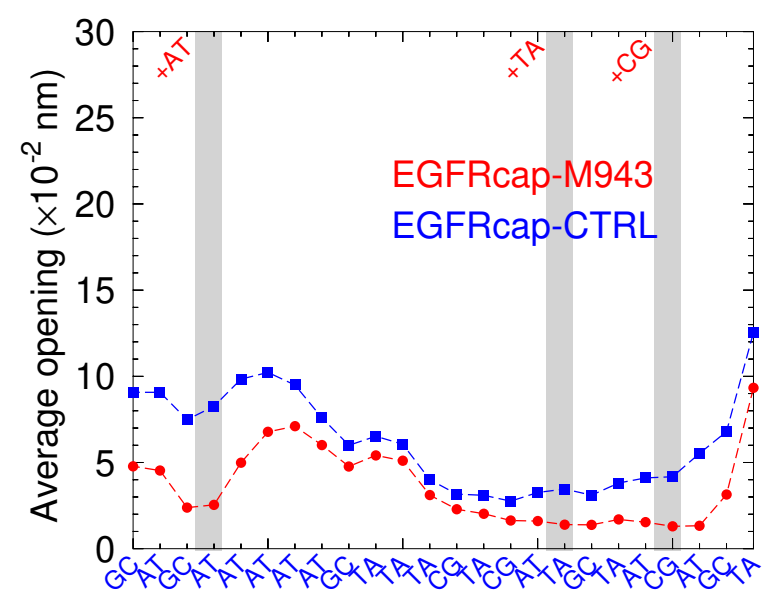

Figure S23: Average opening profile for EGFR capture probe. Modified and non-modified probe are shown in red bullets and blue squares, respectively. LNA modifications are preceded by a plus sign and marked in bold. The location of the LNAs is indicated by the gray shaded area. Calculation was carried out at $220 \mathrm{~K}$ which has no relation to the melting temperature.

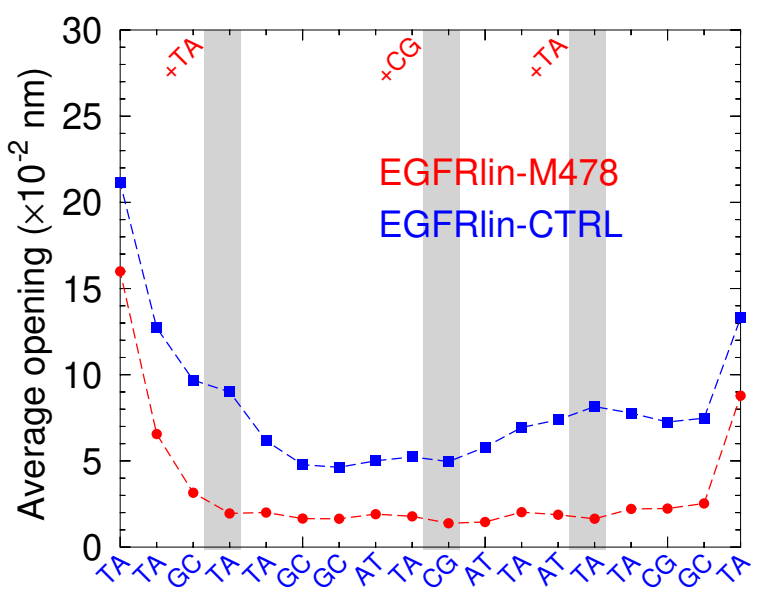

Figure S24: Average opening profile for EGFR linker probe. Modified and non-modified probe are shown in red bullets and blue squares, respectively. LNA modifications are preceded by a plus sign and marked in bold. The location of the LNAs is indicated by the gray shaded area. Calculation was carried out at $220 \mathrm{~K}$ which has no relation to the melting temperature. 


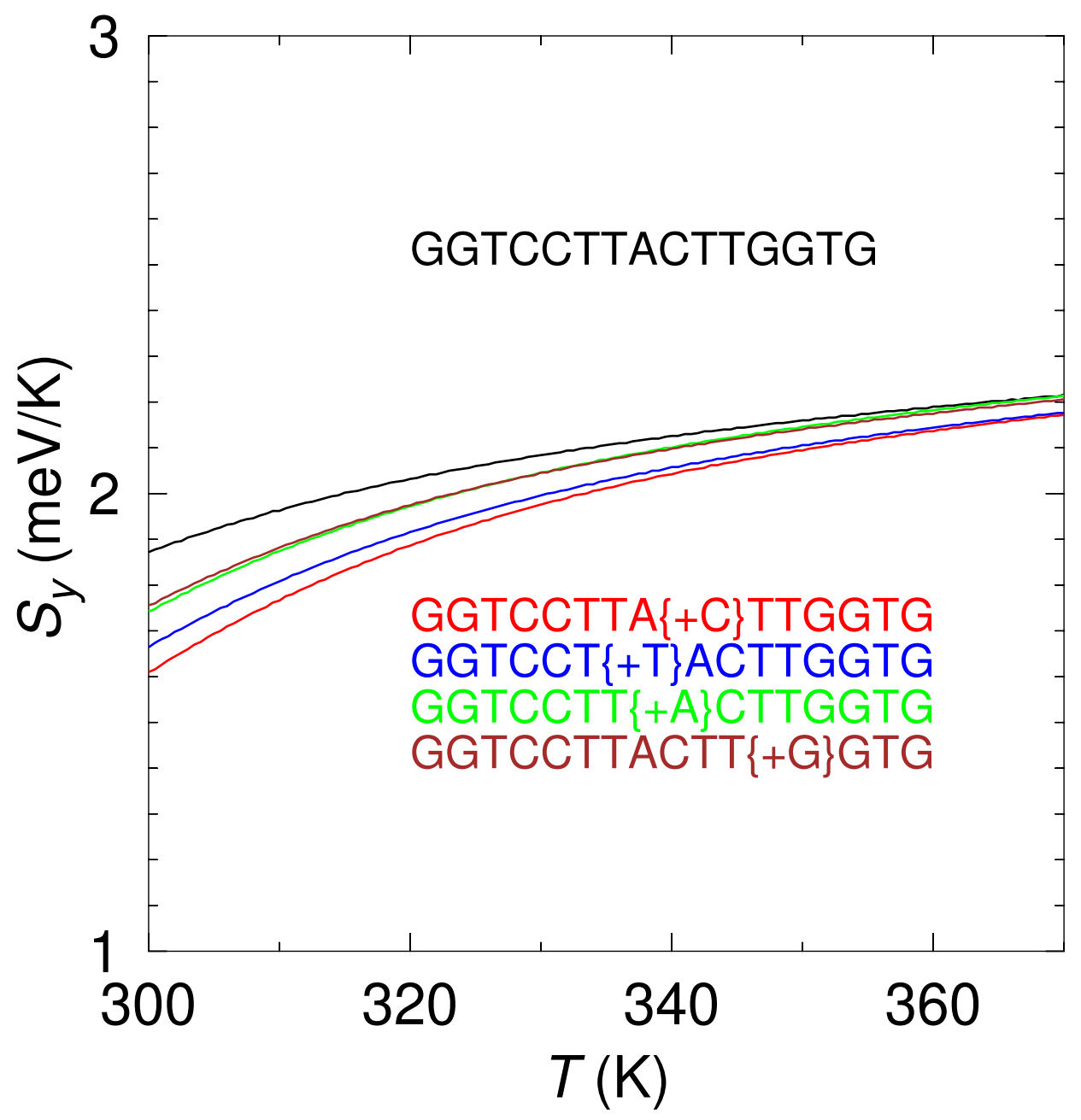

Figure S25: Calculated configurational entropy $S_{y}$ comparing an unmodified DNA sequence (black curve) to LNA modifications $+\mathrm{C}$ (red), $+\mathrm{T}$ (blue) $+\mathrm{A}$ (green) and $+\mathrm{G}$ (brown). The entropy is calculated from the first derivative of the Helmholtz free energy $S_{y}=-\partial F_{y} / \partial T$, which in turn is obtained from $F_{y}=-k_{B} T \ln Z_{y}$, where $Z_{y}$ is the configurational partition function Eq. (2) of the main text. 


\section{References}

[1] Patricia M McTigue, Raymond J Peterson, and Jason D Kahn. Sequence-dependent thermodynamic parameters for locked nucleic acid (LNA)-DNA duplex formation. Biochem., 43(18):5388-5405, 2004. doi: 10.1021/jp073198j.

[2] Yong You, Bernardo G Moreira, Mark A Behlke, and Richard Owczarzy. Design of LNA probes that improve mismatch discrimination. Nucleic Acids Res., 34(8):e60, 2006. doi: 10.1093/nar/gk1175.

[3] Kareem Fakhfakh, Olivia Marais, Xin Bo Justin Cheng, Jorge Real Castañeda, Curtis B Hughesman, and Charles Haynes. Molecular thermodynamics of LNA:LNA base pairs and the hyperstabilizing effect of 5'-proximal LNA:DNA base pairs. AIChE J., 61(9):2711-2731, 2015. doi: 10.1002/aic.14916.

[4] Curtis B Hughesman, Robin FB Turner, and Charles A Haynes. Role of the heat capacity change in understanding and modeling melting thermodynamics of complementary duplexes containing standard and nucleobase-modified LNA. Biochem., 50(23):5354-5368, 2011. doi: 10.1021/bi200223s.

[5] Richard Owczarzy, Yong You, Bernardo G. Moreira, Jeffrey A. Manthey, Lingyan Huang, Mark A. Behlke, and Joseph A. Walder. Effects of sodium ions on DNA duplex oligomers: Improved predictions of melting temperatures. Biochem., 43:3537-3554, 2004. doi: 10.1021/bi034621r. 